\title{
From Automation to AUTONOMY - A NeW Trend for SMart MAnUfaCtUring
}

\author{
PARK, H-S.
}

Abstract: Today's dilemma of the manufacturing system is to realize stable processes while manufacturing's environment is affected by global competition, high degree of complexity and autonomy, short delivery times and high product variety. In order to guarantee the requested product quality and reduce downtime, the smart manufacturing (SM) which is considered as a new trend helps to meet objectives associated with these problems and improve the productivity, quality, cost and competiveness. From an engineering perspective, the SM is the reinforced application of advanced intelligence and cognitive technologies to enable rapid productivity, dynamic response to product quality demand and real-time self-optimization of production process. It creates an autonomy environment across a series of workpiece and manufacturing system. Nowadays, due to the evolution of the information and communications technologies, the SM technique has promised to explore selfoptimizing manufacturing in industry by its potential such as maintaining process stability. For this purpose, in this article the benefits and challenges of self-optimizing manufacturing concept regarding its capability and responsibility are presented describing the adaptation to changing manufacturing environment.

Key words: autonomy, smart manufacturing, self-optimizing processes, adaptability, intelligence
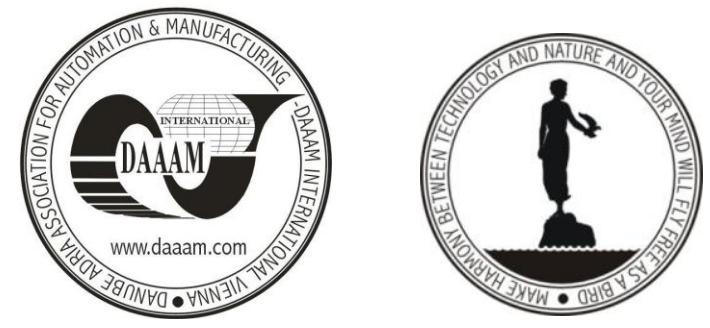

Authors' data: Univ.Prof. Dipl.-Ing. Dr Park, H[ong-Seok]; Daehak-ro 93, Nam-gu, Ulsan 680-749, South-Korea, phosk@ulsan.ac.kr

This Publication has to be referred as: Park H[ong-Seok] (2013) From Automation to Autonomy - a New Trend for Smart Manufacturing, Chapter 03 in DAAAM International Scientific Book 2013, pp. 075-110, B. Katalinic \& Z. Tekic (Eds.), Published by DAAAM International, ISBN 978-3-901509-94-0, ISSN 1726-9687, Vienna, Austria

DOI: $10.2507 /$ daaam.scibook.2013.03 


\section{Introduction}

Manufacturing systems of the future will be characterized by the strong individualization of products under the conditions of value-added processes and high quality services. So, new technologies and methods are researched for the next stage of industrial manufacturing. Numerous researches in the manufacturing field to achieve an intelligent manufacturing have been reported in the literature as shown in Fig.1. The research area can be classified as follows:

- Technologies for the advanced information systems such as manufacturing execution system (MES) and process planning, industrial network, inheritance of data and information and communication technology (ICT) for industry.

- Evolvable hardware/software such as integration of industrial systems, intelligent diagnosis, effective maintenance for equipment and system, hitech machinery industry and intelligent sensors.

- Manufacturing system architecture such as international standards, design technology and model of the manufacturing system.

In recent years, due to the hard competitiveness through globalization most manufacturing companies have focused on manufacturing higher value added products with low production costs. So, it is inevitable to apply the advanced technology to carry out it in effective and efficient way.

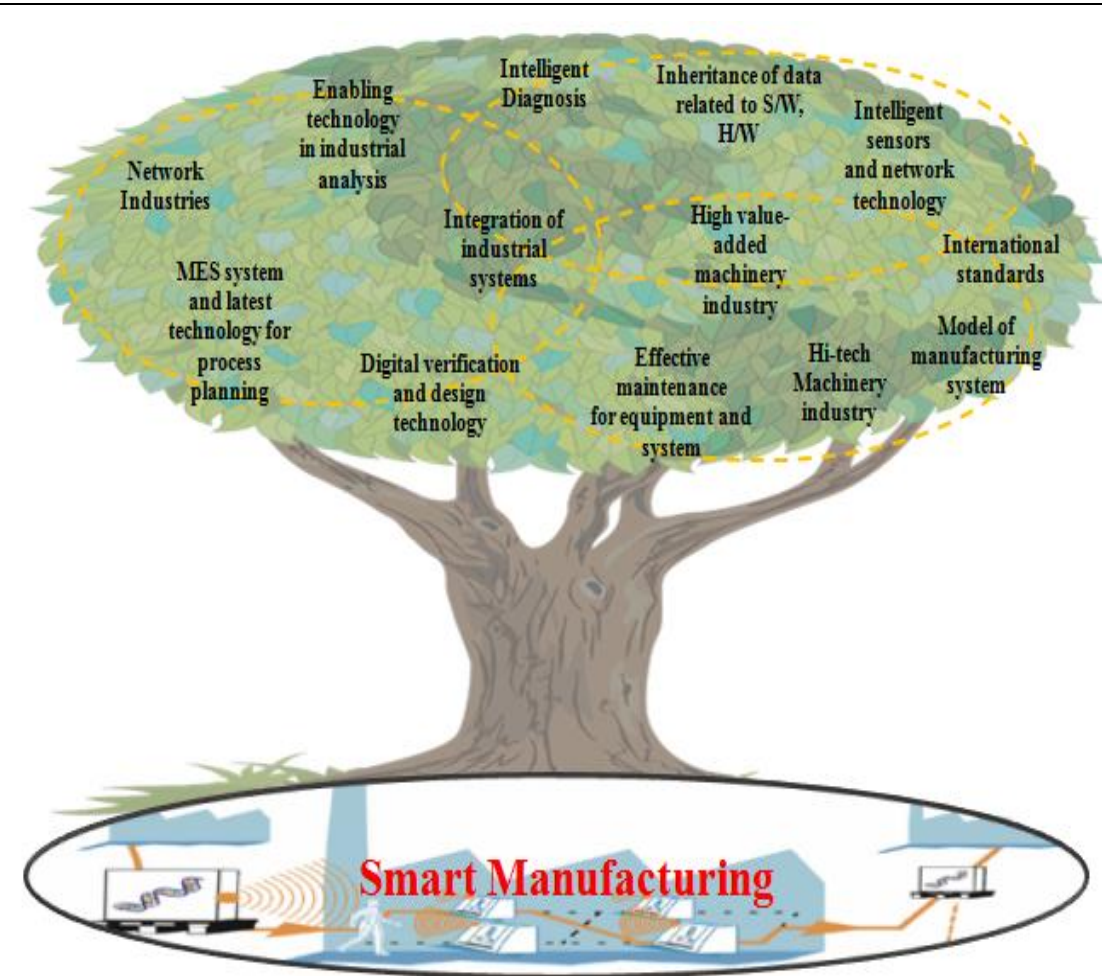

Fig. 1. Research areas in intelligent manufacturing 
Monitoring and control of machining processes are becoming increasingly important for keeping a consistent quality of machined parts.

The article presents in-process sensing and control techniques with applying information science for the next generation of quality control that eliminates the need for expensive post-process inspection. In this research, a machining process of machine tool is considered in terms of a quality oriented control. The self-optimizing control is proposed for enabling intelligent process monitoring and control which can detect the cutting conditions and status of machining operations. To prove the proposed self-optimizing control system, self-monitoring and self-adjusting, the machining process related to the tool condition such as tool wear was considered in this article.

The adaptation of a manufacturing system to disturbances is abilities to respond rapidly and to recover autonomously, which keep the manufacturing system running and avoid stopping completely. Autonomy allows the system to recover autonomously. The article presents an autonomous manufacturing system (AMS) where all resources of a manufacturing system are modeled as intelligent entities. Each entity has abilities of identification, data collection and making a decision autonomously. The AMS for adapting to disturbances brings the following advantages:

- Allowing the control system to take action during disturbance and to continue to operate instead of stopping the manufacturing system completely

- Equipping entities in the manufacturing system the decision making and self-controlling abilities

The new trend of the manufacturing system development is to apply autonomous behaviours inspired from biology for the manufacturing systems. Intelligent entities of the AMS equipped cognitive capabilities are necessary, but they are not enough. The AMS implemented needs not only intelligent entities but also an autonomous control architecture of the manufacturing system. The control architecture is changed from the centralized control of non-intelligent entities in hierarchical structures towards the decentralized control of intelligent entities in distributed structures.

\section{Core Technologies for Realizing Smart Manufacturing}

\subsection{Cognitive Technology}

The vision of cognition based manufacturing is shown in Fig.2. In which the intellectual functions such as autonomy, learning, planning, and interaction are equipped with manufacturing equipments and processes in order to improve intelligent, adaptive, reliable, agile, and responsive characteristics of the system. Wireless environment ensures the efficiency in communication and cooperation among machines, devices and sub-systems in the factory. In cognitive manufacturing 
systems, two aspects of cognition, i.e. communication and intellectual functions as shown in Fig.3, are considered to apply into manufacturing environments.

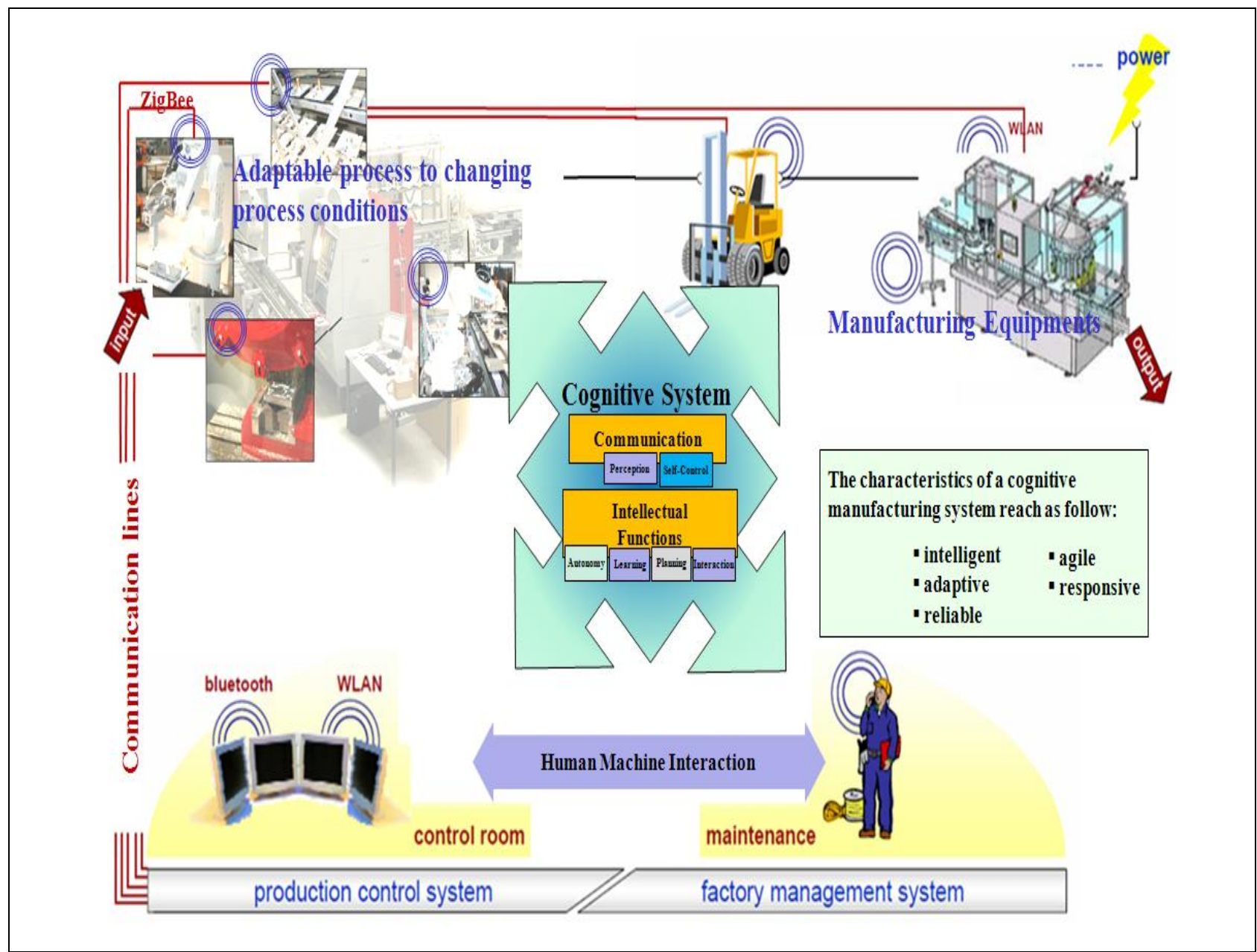

Fig. 2. The model of cognition based manufacturing

The first aspect considers methods for data acquisition, observation; connecting machine to machine, machine and human. The second aspect is intellectual functions such as learning, reasoning, and control. The cognitive system was proposed by Zhao and Son (Zhao \& Son, 2008). In this system, the BDI architecture inspired from cognitive science was applied. Beliefs are information about the shop floor such as the status of machines and processes. Desires are all the possible states of tasks that the system would like to accomplish. Intentions are the states of the tasks that the system has decided to work towards.

This architecture is based on a human decision-making model from cognitive science that comprises knowledge models, methods for perception and control, methods for planning, and a cognitive perception-action loop.

The paradigm "cognition" in terms of the manufacturing system denotes that machines and processes are equipped with cognitive capabilities and cognitive controls in order to enable them to increase their scope of autonomous operations. A cognitive control consists of three general actions: perceiving information in the 
environment, reasoning about those perceptions using existing knowledge, and acting to make a reasoned change to the environment. Cognitive capabilities such as perception, reasoning, learning and planning turn the technical system into ones that is "know what they are doing" (Zaeh et al., 2009). Manufacturing systems with cognitive capabilities will be much easier to interact and cooperate with, and they will be more robust, flexible and efficient.

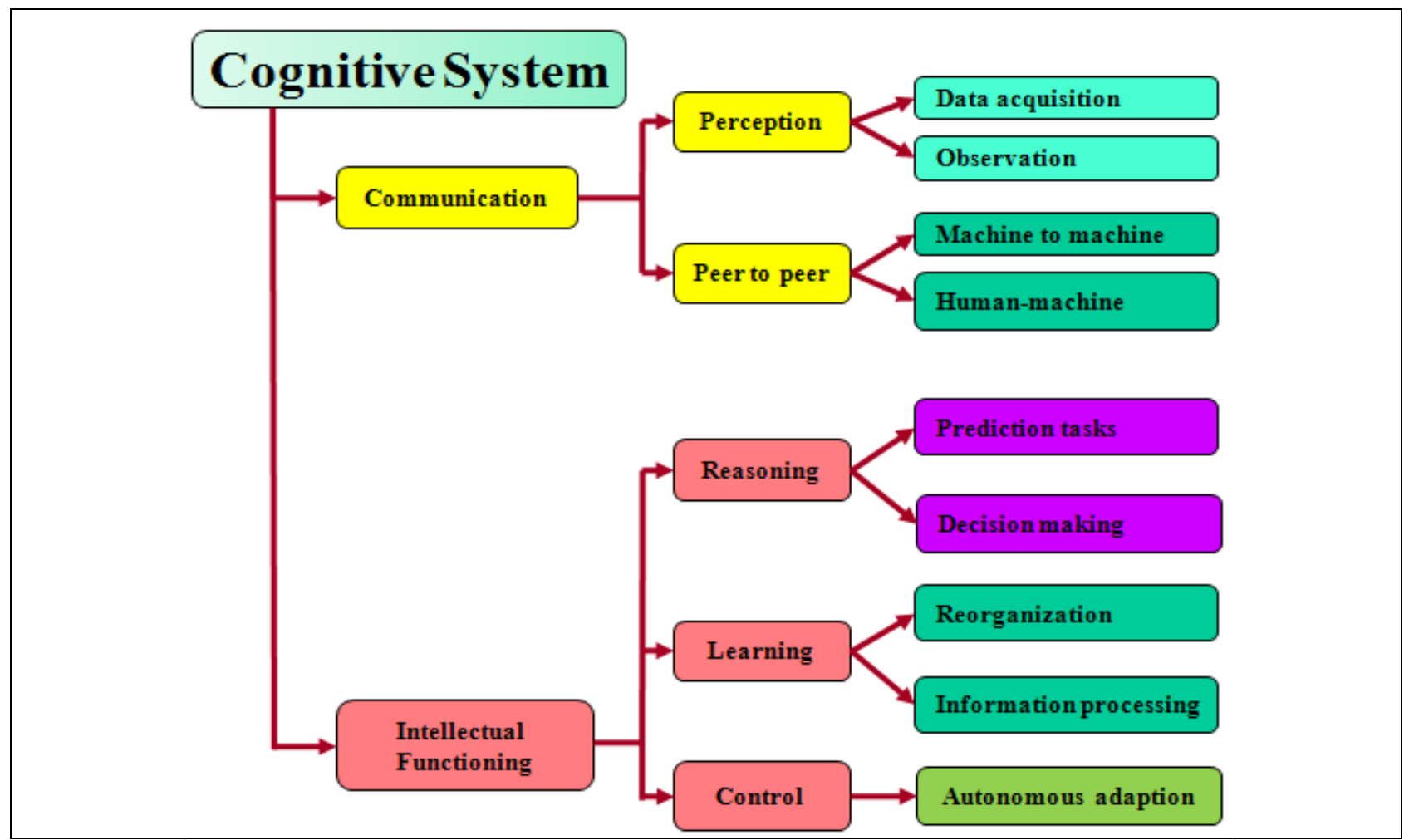

Fig. 3. Cognitive system

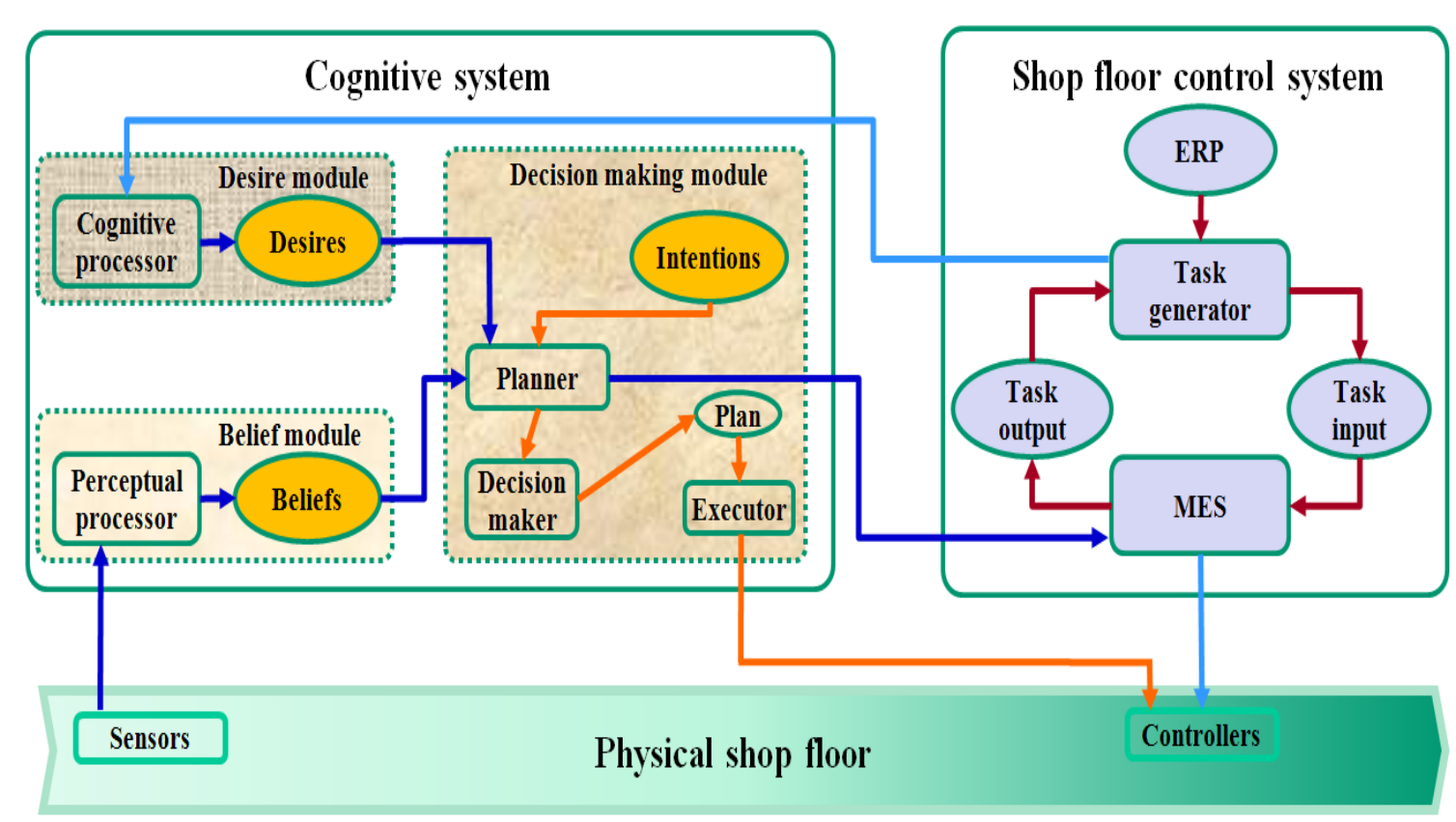

Fig. 4. Cognitive system for controlling a shop floor 
Fig.4 illustrates the cognitive system for controlling the shop floor. This framework is based on the BDI architecture of human decision making. In this framework, the cognitive system is responsible for monitoring and controlling the shop floor. The task generator receives orders from the ERP and then generates sequences of tasks which are required to produce the orders. These tasks are sent to both of the MES and the cognitive system. The belief module is responsible for updating information on the shop floor. These data are then compared with the desires in the desire module by the decision making module. In case the data match with the desired goals, a message is sent to the MES to report the normal status and the shop floor continues running. Otherwise, the data are different from the desired goals because of an error happening. The decision module generates a new plan and controls the shop floor. The MES controls the shop floor when the machines or processes are recovered.

Learning can be defined as a way to acquire knowledge and skills to adapt the changes, and it is crucial to respond to the dynamic of environment and to improve the system ability to act in the future by taking a better decision and performing better the required actions (Brezocnik et al., 2003). Learning is normally performed in result of the decision making process allowing to adjust the decision parameters or to update the behaviour rules. In the manufacturing control context, the learning mechanisms are triggered mainly in the situations: the process finishes, the system configuration changes, and unexpected disturbances occur. Reasoning is used in prediction tasks and decision making. Several reasoning mechanisms are used such as causal, temporal, and spatial reasoning which enables the system to solve dynamically changing and more complex tasks. Reasoning plays the main role for autonomous adaptation process of the system where the system adapts to disturbances and changes autonomously.

\subsection{Agent Technology}

Agents can be classified such as biological agents (human), robotic agents (autonomous robots), and software agents as shown in Fig.5. In this research, the agent technology is applied to implement distributed systems by set of software agents. Software agents are computer programs that have characteristics such as autonomy, social ability, reactivity, and pro-activeness (Leitao, 2009a).

The autonomy is an ability of the agent to achieve its goals without any support from the other agents. On the other hand, the interaction between agents is the social ability of agents that is used to get the global goal of the system. The reactivity, which is based on the relation between perception and action, is an ability of agents to respond to the environmental changes. The pro-activeness of agents is an ability to express the goal-directed behaviours. 


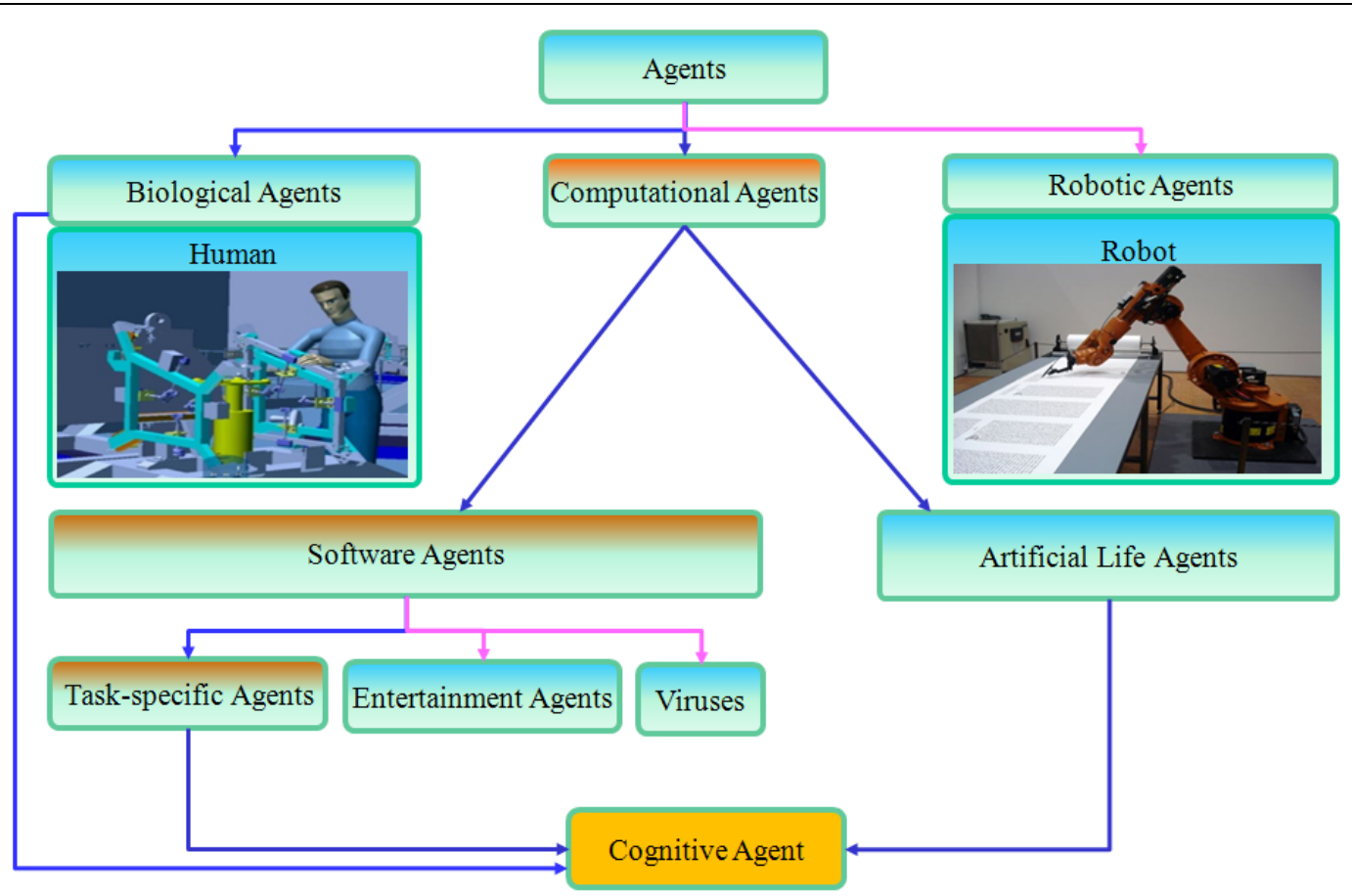

Fig. 5. Agent classification

With the conventional agent, the reactions of agent to changes of the environment (status of the machine tool) are based on the rules. The mentioned characteristics of the agents are necessary to develop the distributed control system of the AMS. In practice, many applications based on the agent technology have been proposed to deal with disturbances by dynamic rescheduling and reconfiguration. This method is an effective way to overcome disturbances which are long time to recover or unable to recover. However, in case disturbances require a short time to recover, agents for the self-recovery system without rescheduling are more suitable than a total scheduling modification.

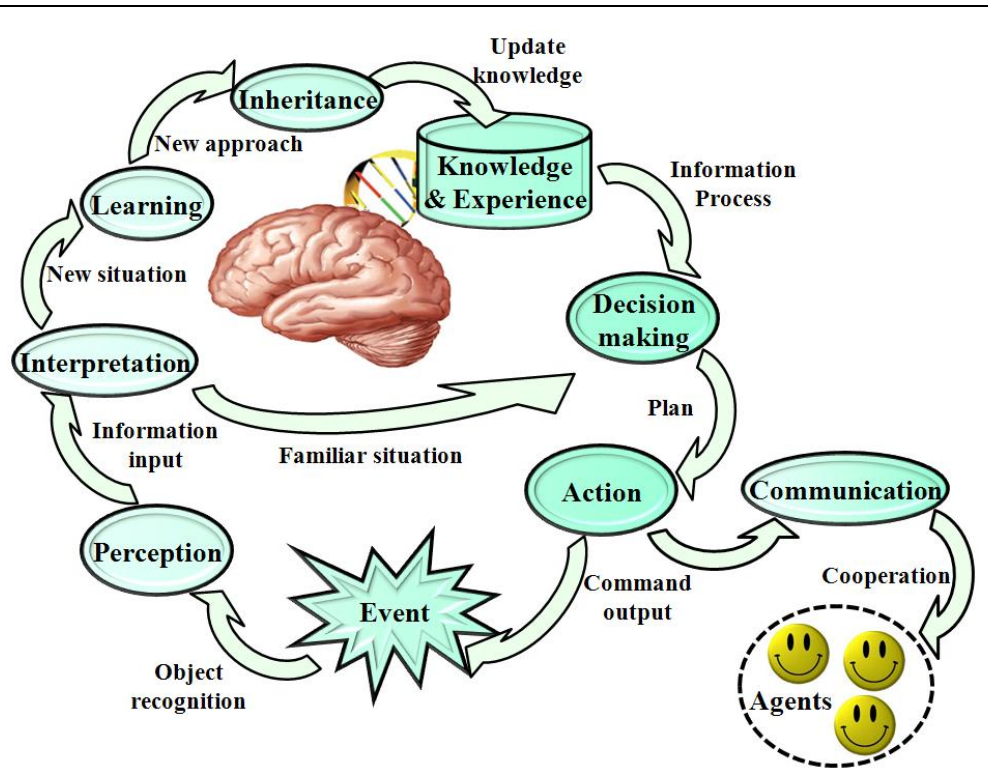

Fig.6. Architecture of cognitive agent 
The reactions of agents to the environmental changes are the reactivity or proactiveness that depends on what kind of agent architecture is applied to develop the agents.

These software agents are further divided into task-specific agents, entertainment agent, and viruses. In the manufacturing field, the task-specific agent is improved their capabilities in terms of scope of autonomous operations by the integration of biological characteristics and artificial life. Artificial life studies the logic of living systems in artificial environments. The goal is to study the phenomena of living systems in order to come to an understanding of the complex information processing. Cognitive architecture is applied to build cognitive agents as shown in Fig.6. Consequently, the agents are equipped with cognitive capabilities such as reasoning to make a decision, and cooperation which emulate human cognitive behavior.

\subsection{Cognitive Agent}

A synthesis approach of the agent and cognitive technologies are applied to improve the autonomous characteristics of conventional agents. As the result, a smarter agent, namely cognitive agent, is proposed. The cognitive agent is a computer program equipped with artificial cognitive capabilities in order to perform the cognitive activities which emulate the cognitive behaviours of human such as perception, reasoning and decision making, communication, and learning. Cognitive agents ensure the flexibility of the manufacturing system for adapting to the changes and unexpected disturbances. The cognitive agents implement a cognitive perceptionaction loop that does not only adapt to the changes of manufacturing system autonomously but also inherits any new optimal plan generated for adapting to disturbances. Fig.6 shows the architecture of a cognitive agent for processing events occurred from the resource controlled by the cognitive agent such as tool-wear, machine breakdown and so on. The perception module is responsible for a data acquisition from the resource through sensors. This data observation includes many kinds of data such as visual data, auditory data, vibration data, and so on. The interpretation module is responsible for transforming these data to the standard format that describes the status of the resource. The cognitive agent uses its own knowledge and experience to make a decision that is suitable for the status of the resource. In order to cope with an unfamiliar status, self-learning and inheritance capabilities are equipped for the agent. AI techniques are applied to generating a new solution for adapting to the unexpected status of the resource, e.g. machine learning techniques adapting to dynamic changes of the environment. The cognitive agent makes a decision like a new plan based on the updated knowledge to cope with the resource changes. The action module processes this plan into tasks and executes the tasks at the resource. At the same time, this module sends the tasks to the communication module if the plan is processed by the agent team.

\subsection{Swarm Intelligence}

In the natural environment, a collective intelligence is carried out by simple interactions of individuals. A concept found in the colonies of insects, namely swarm intelligence, exhibits this collective intelligence (Leitao, 2009b). Swarm intelligence 
is established from simple entities which interact locally with each other and with their environment such as colonies of ants, schools of fishes, flocks of birds, and human cooperation as shown in Fig.7. Swarm mechanism is applied to the manufacturing field. In ant colonies, the collective intelligence is given by interactions of individual ants with the limited cognitive abilities expressed by chemical substances called pheromones. One of the collective intelligence of an ant colony is finding the shortest route from the nest to the food source. The first ant finds the food source and returns to the nest leaving behind a pheromone trail. Over time, the evaporation of the pheromone trail begins to reduce its attractive strength. The pheromone evaporation is a criterion for avoiding the convergence to a locally optimal solution. Ants can follow many possible ways from the nest to the food source and back again, but the strengthening of the route making them more attractive is the shortest route (Leitao, 2009b; Garg et al., 2009).

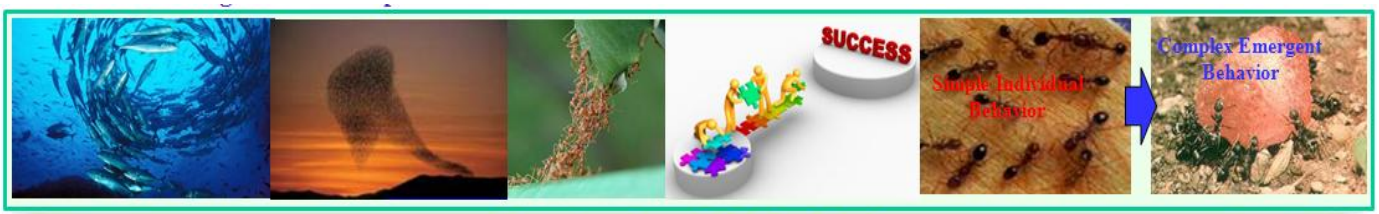

Bio- Inspired Technologies

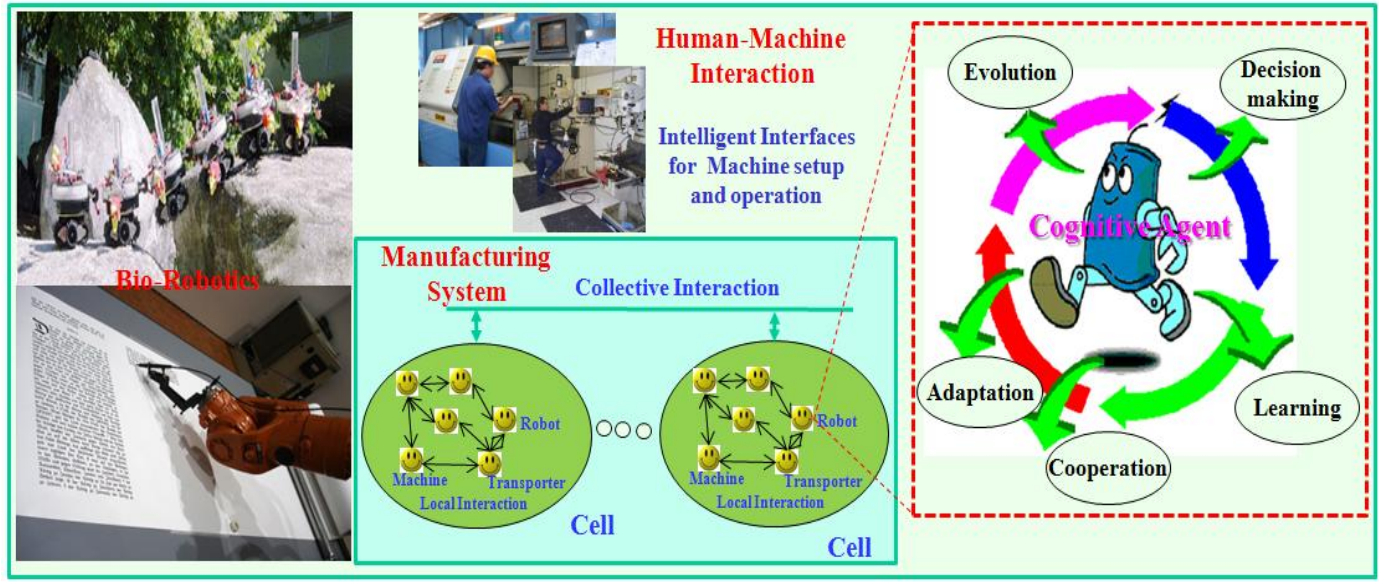

Fig. 7. Bio-inspired technologies for manufacturing

Transferring this principle to the manufacturing field, most current manufacturing applications such as manufacturing scheduling and manufacturing control use the swarm intelligence technology. In which, swarm intelligence technology is expressed either as evolutionary algorithms (ant colony optimization, particle swarm intelligence) (Anghinolfi, et al., 2007) or as a multi-agent system (Leitao \& Restivo, 2002). In order to adapt with the dynamic evolution of environment, a swarm of ants needs the self-organization ability. Self-organization is carried out by re-organizing its structure through a modification of the relationships among entities without external intervention. In manufacturing systems, which is seen as a community of autonomous and cooperative entities, self-organization is carried out by locally matching between machine capabilities and product requirements (Nakano \& Suda, 2007). Each machine has a pheromone value for a 
specific operation and the machine with the shortest processing time for a specific operation has the highest pheromone. A machining process planning provides information on how to machine the designed products. This information includes machining operations and parameters needed for machining to convert a part from a raw material to a given shape. The machining process planning is automatically generated by using the CAPP system. Most of the current available CAPP systems are centralized in architecture and off-line data processing. Even though AI techniques are applied to generate an optimal process planning, the conventional CAPP systems are difficult to make adaptive decisions in advance, without knowing actual the status of machines on the machining shop floor. The new pursuit for generating a machining process planning is to apply cognitive agent technology. In this method, work-piece, machine tools, robot, transporter, and so on are controlled by the corresponding cognitive agents.

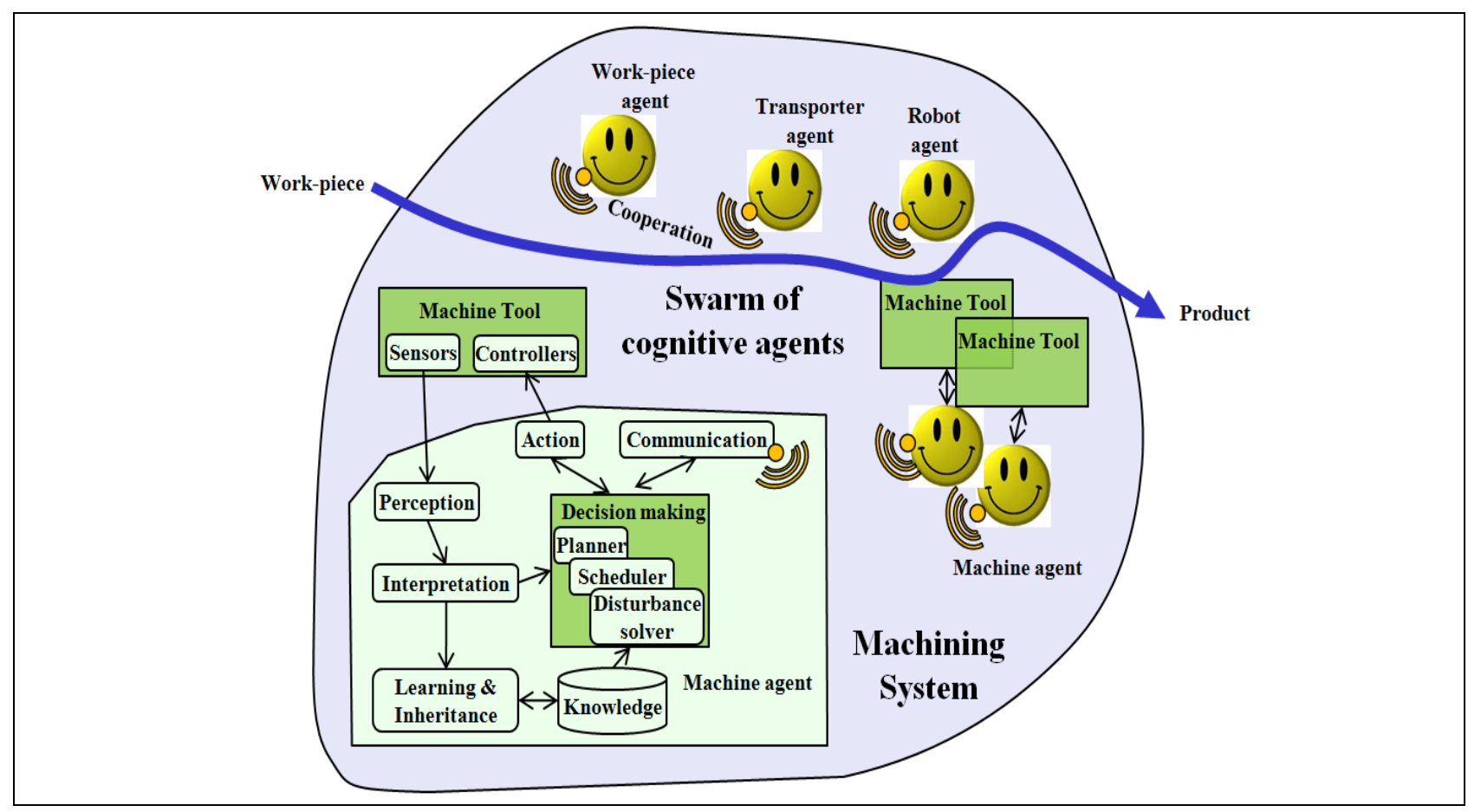

Fig. 8. Swarm of cognitive agents for the machining system

The machining system is considered as a swarm of cognitive agents shown in Fig.8, in which the whole machining process planning for the designed product is generated by the cooperation among cognitive agents. Each machine agent has the ability to generate a process plan for itself and negotiate with other agents in order to carry out machining process in effective and efficient way. The negotiation ensures that all tasks are allocated to the corresponding machine agents, consequently, avoiding conflicted tasks among these agents.

\subsection{ICT Infrastructure}

In communication, resources of the manufacturing have the ability of identification, data collection and making a decision autonomously due to an ICT infrastructure. The ICT infrastructure contributes significantly to the success of 
implementing the autonomous manufacturing system (AMS). The MES provides an interface between an ERP system and shop-floor controllers for executing the functionalities such as scheduling, order release, quality control, and data acquisition (Choi \& Kim, 2002; Kletti, 2007; Valckenaers et al., 2007). The ERP system supports almost all business systems whose single database is used for business functions such as production and supply chain management. The ERP system also can be considered as the informational backbone of IT infrastructure for manufacturing system.

Radio frequency identification (RFID) technology and the sensor technology related to it have a great potential in changing the way of control, production automation, and special data collection in connection with the higher level such as ERP, supply chain management (SCM), and customer relationship management (CRM) (Günther, et al. 2008) as shown in Fig.9.

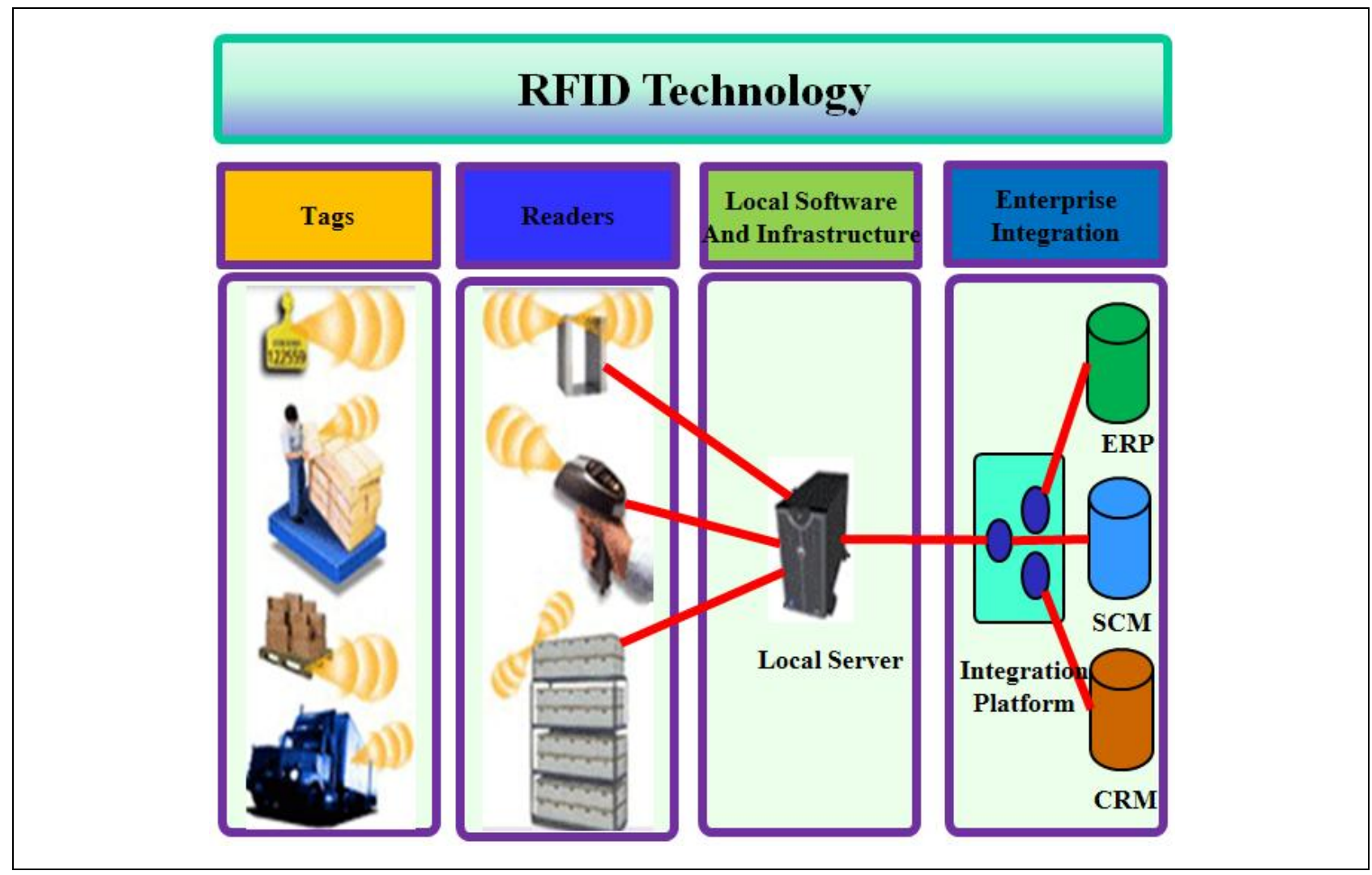

Fig. 9. RFID technology in manufacturing

They also make a contribution for cutting down labor cost, reducing breakdown time, and improving production effectiveness. In the machining system controlled by the cognitive system, RFID technology plays the role of tracking on core components in complicated processes in real time because this technology enables to read and write data to an RFID tag at the moving parts.

Ubiquitous sensor network (USN) is a tool for collecting production data in realtime constraint. The USN plays the role of monitoring for machine operating status, actual production, and increasing the product quality (Kim, et al., 2009). A 
component is called intelligence if it shows advanced characteristics, such as unique identification, communication with the environment, ability to store data about itself, language to display its features or production requirements (Meyer et al., 2009). Currently, the intelligent components use RFID tags for storing information. The tags are attached to the machine components which may be detached occasionally during the manufacturing process. As it is inspired from the biological organism of which the information exists within itself, a new approach is to store directly the production data on the component surface by merging the information and component (Denkena et al., 2008; Schmidt et al., 2008). For the chosen approach, it is necessary to develop the magnetic magnesium $(\mathrm{Mg})$. The $\mathrm{Mg}$ used as a sintered material is integrated into an appropriate component (Wu et al., 2008; Wu et al., 2010). The vision of "feeling" machine components is achieved by attaching multi-sensor system to these components (Denkena et al., 2010). Intelligent components are the results of applying sensor technologies and the ICT progress that ensure the precise operations and flexibility of the manufacturing system.

Ubiquitous computing, ubiquitous networking and ambient intelligence are three representative conceptions that embody the most important aspects of the ubiquitous technology. In ubiquitous computing, the computing devices are embedded into the machine tools so that the operators can interact with the devices at the same time as they interact with the machine tool. In ubiquitous networking, computers and machines can be operated at any place, at any time. Ambient intelligence comes out from the integration of ubiquitous computing and ubiquitous networking. Normally, it represents a well defined space with a certain level of intelligence that results from embedding technology (Serrano \& Fischer, 2007).The model of ubiquitous machine (U-Machine) is shown in Fig.10. Being ubiquitous machine, the machine status and machining data of $\mathrm{CNC}$ machines can be monitored with wired/wireless environments, including the environments of international mobile telecommunications-2000 (IMT2000) and wireless LAN. CNC machines are controlled and monitored in real-time, anywhere and anytime. Moreover, prompt notification from CNC machines to mobile phones is automatically realized in emergencies.

Cloud computing is the next step in the evolution of ICT infrastructure as shown in Fig.11. It enables to realize collaborative design, distributed manufacturing, collective innovation, data mining, semantic web technology, and virtualization. In smart manufacturing, cloud computing enables to develop products quickly with minimum costs through a social networking. Model of the cloud computing system is parallel and distribution which consists of a collection of inter-connected physical and virtualized service pools of design and manufacturing resources. The manufacturing cloud service can offer capabilities for design and manufacturing solutions at certain levels, such as manufacturing cells, general purpose machine tools, and standardized machine components (Wu et al., 2012). 


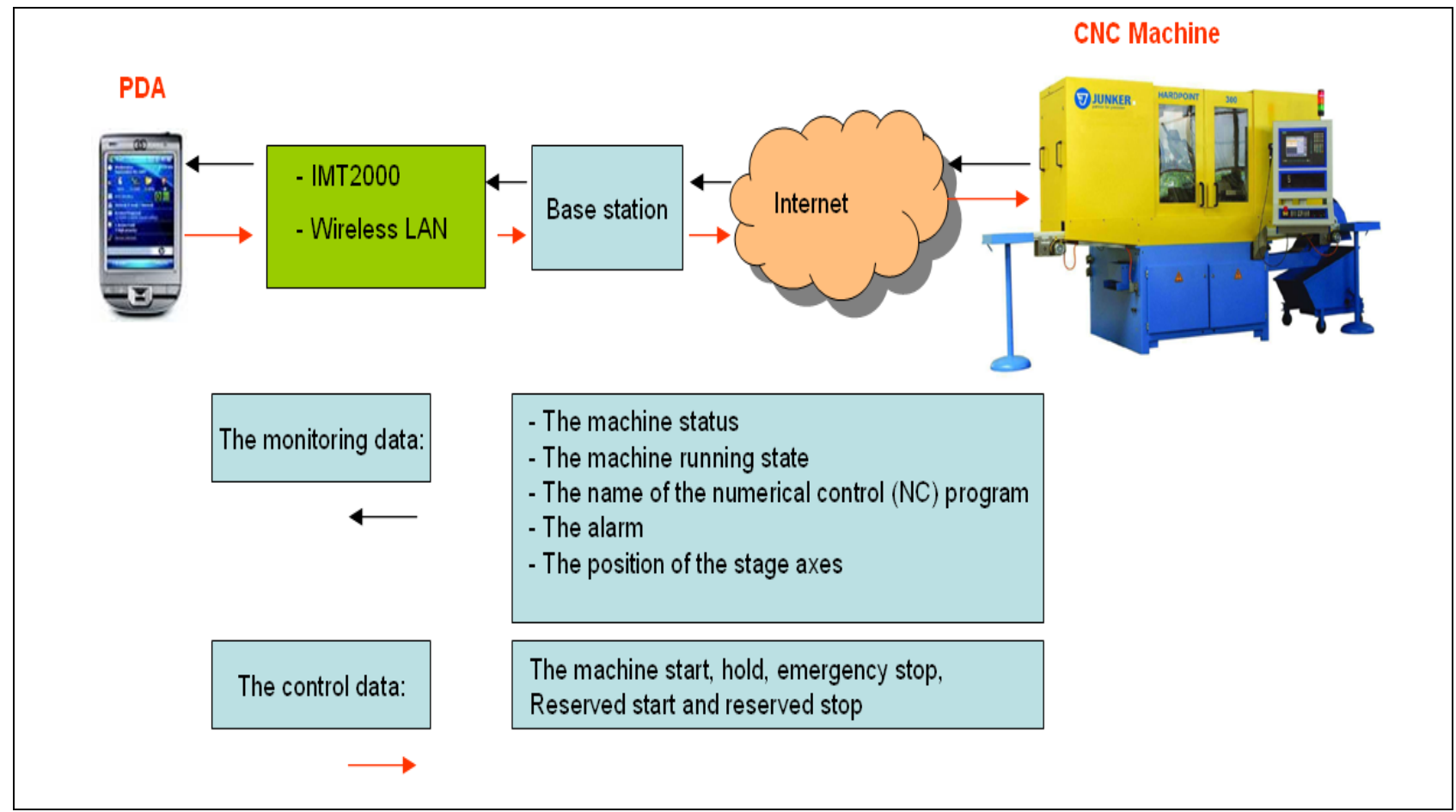

Fig. 10. Model of U-Machine

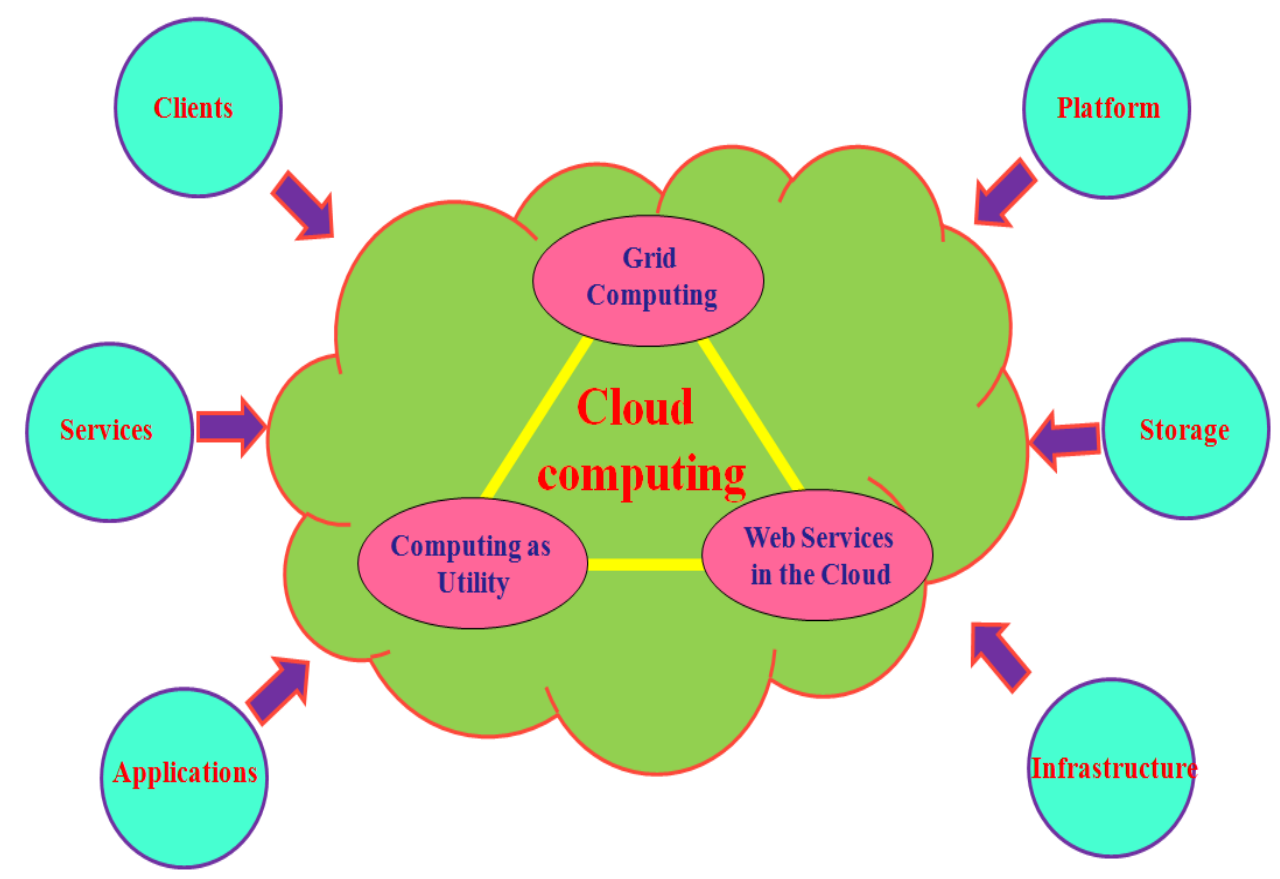

Fig. 11. Concept of cloud computing

\section{Characteristics of Smart Manufacturing}

\subsection{Adaptability}

Adaptation to changes of manufacturing environment is a crucial issue in the development of smart manufacturing systems. It is the ability of a manufacturing system to respond rapidly to disturbances to keep the manufacturing system running and avoid the manufacturing processes stopping completely. Reconfigurable 
manufacturing system (RMS) is designed for rapidly adjusting its production capacity and functionality by rearranging or changing its components to adapt to the changes of the manufacturing environment. Unlike the flexible manufacturing system (FMS), the RMS does not have a fixed capacity and functionality, and it is designed through the use of reconfigurable hardware and software. The RMS could be reconfigured both on the overall system's structure level and on the machine level. Machine tools evolved through advances in hardware and control technologies play an important role in the RMS (Shirase \& Fujii, 2009; Yigit, ). Their performance has a high effect on both the performance and productivity of the manufacturing system. The reconfiguration of machine tools is required by changes in manufacturing requirements and market demands. In which the physical hardware reconfiguration as well as logical reconfiguration of machine tools are considered (Pritschow et al., 2009; Mori \& Fujishima, 2009; Abele \& Worn, 2009; Landers et al., 2001).

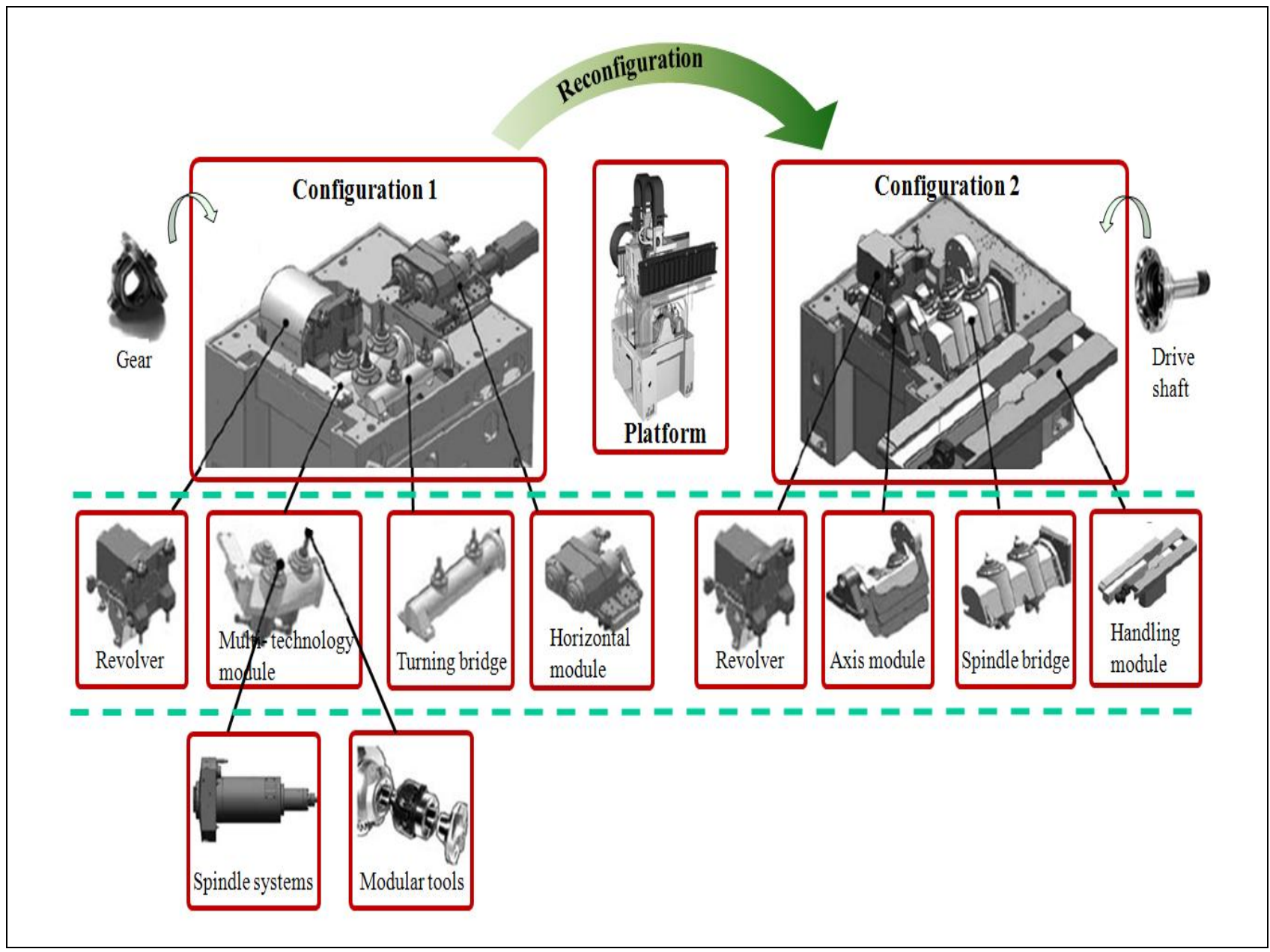

Fig. 12. Modular oriented architecture of the machine tool (Abele \& Worn, 2009)

Nowadays, machine tools are designed as modular systems that ensure to match the respective applications by reconfiguration (Yoshimi, 2008) as shown in Fig.12. Technical innovation in hardware of machine tools has been shown by generation of turning and milling integrated machine tool, five-axis machining center, and ultraprecision machine tool. The main characteristics of this generation are the change of function and structure of the machine tool to perform multi-tasks and multi-axis. The 
machine tool needs the module-oriented architecture as well as the self-adapting control system for reconfiguration. Consequently, besides the development of the functional and structural machine tool, the innovation in control systems toward intelligent machine tools has been studied. As the result, the self-adaptive control has been proposed as the current technology of advanced computer numerical control (CNC) machine tools. The self-adapting control consists of the following elements:

- A modular and open system architecture allowing the exchange, integration and interconnection of software components.

- Control software components implementing the mechanisms for monitoring and identification of mechanical components.

- A mechatronic integrating model for the description of the machining modules as well as for the control software components and the relations between them.

- An integrated methodology for the reconfiguration process based on the actions undertaken by a human control system specialist.

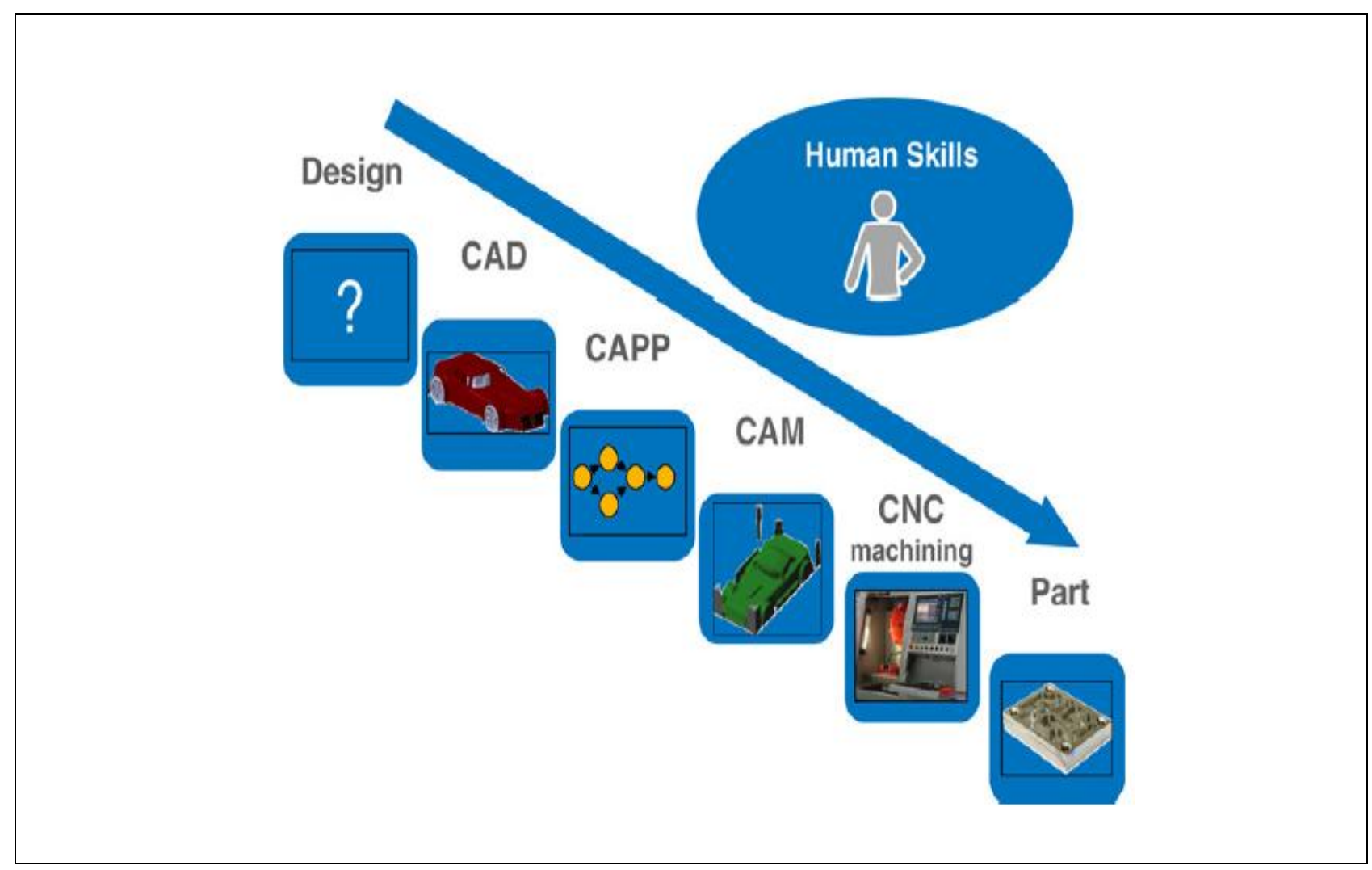

Fig. 13. Current design-to-manufacturing process

\subsection{Intelligence}

The human beings can adapt to the environmental changes by the cognitive capabilities such as perception and intellectual functions in which the learning capability allows the human being to improve the knowledge and skill to adapt to the changes. Currently, human workers with their skills and knowledge adapt to changes of almost all activities from design to manufacturing process as shown in Fig.13. The new trend is to apply the decision capability, knowledge, and human being 
capabilities into the manufacturing system that shows the combination of cognitive science, automation technology, and computer science as shown in Fig.14.

\subsection{Autonomy}

The conventional manufacturing systems, such as FMS, computer integrated manufacturing (CIM) are unable to adapt to the complexity and dynamic of the manufacturing environment. These systems activate the automatic operations by using the pre-instructed programs that reduce the flexibility of the systems.

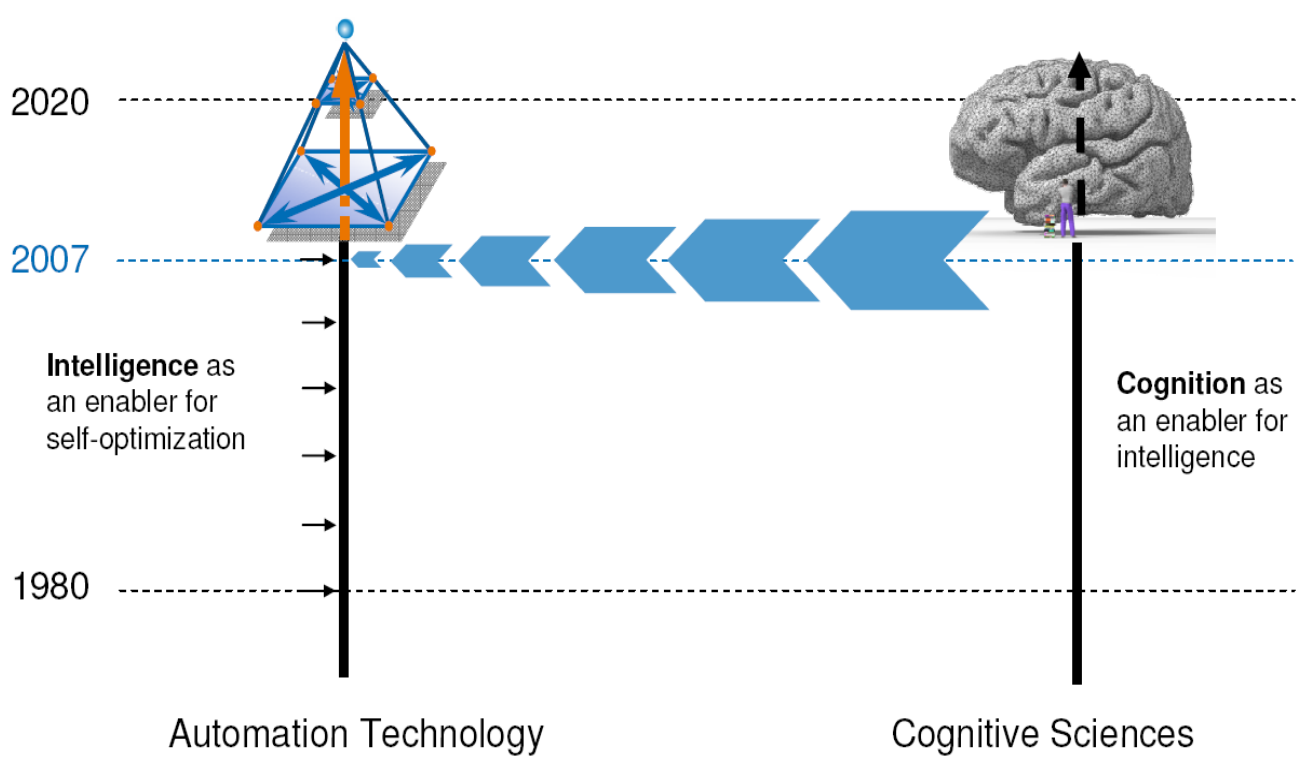

Fig. 14. Cognition for realizing intelligence in manufacturing (Tobias et al., 2009)

The new trend in manufacturing filed is to apply the bio-inspired technologies to equip the machines and processes with autonomous behaviours as shown in Fig.15.

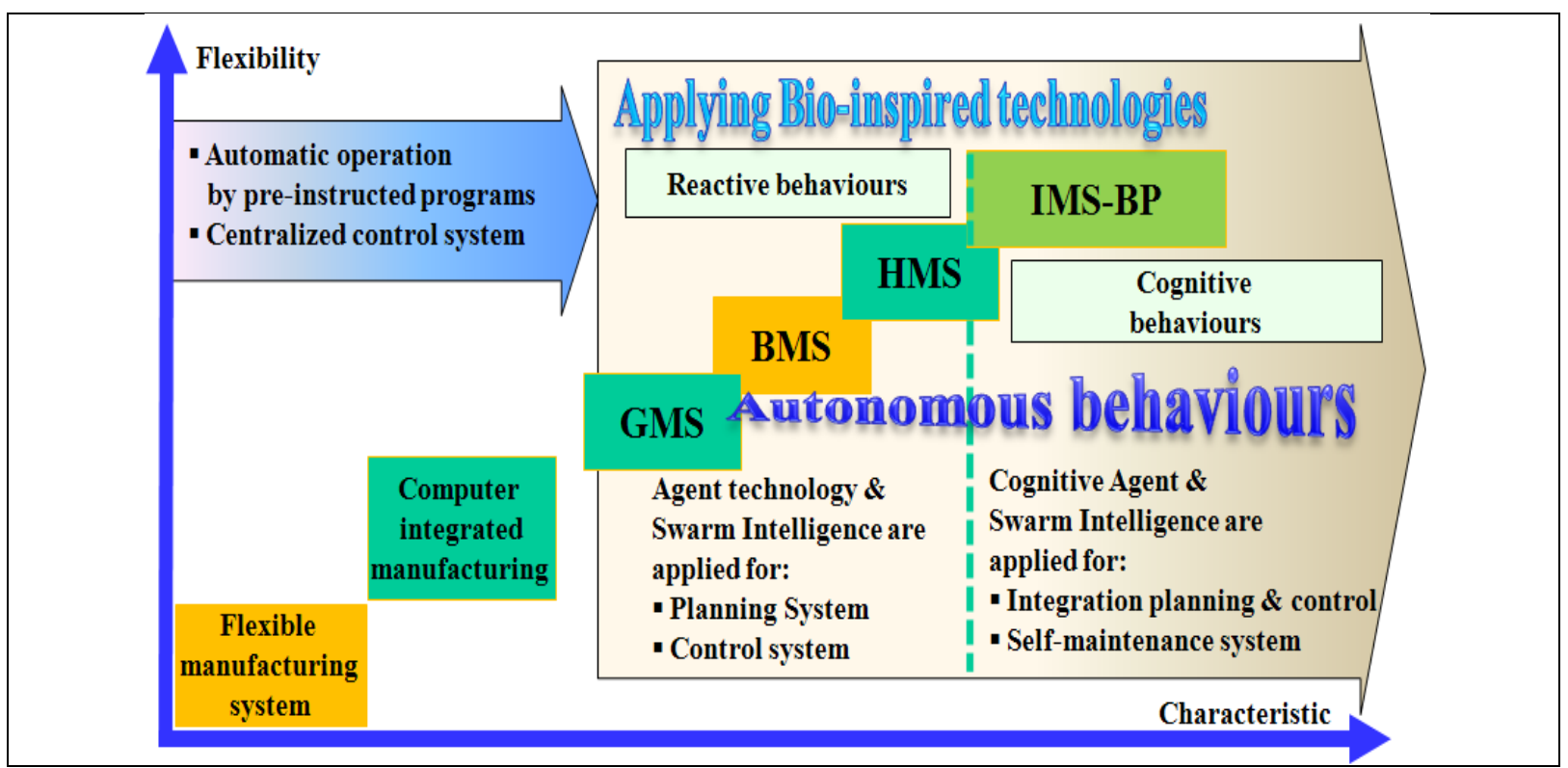

Fig. 15. Evolution of manufacturing systems toward the autonomous manufacturing 
The new concepts in manufacturing have been proposed such as genetic manufacturing system (GMS), biological manufacturing system (BMS), holonic manufacturing system (HMS), and intelligent manufacturing system with biological principles (IMS-BP).Autonomy allows the system to recover autonomously without either upper level aids such as the enterprise resource planning (ERP), and the MES or the operator intervention. The autonomous method is suitable for the changes that are necessary to reschedule. In this method, each entity in the manufacturing system is an autonomous entity so that it can overcome the disturbances by itself or communicate with the others for against disturbances. Reactive and collaborative methods were proposed following this criterion (Monostori, et al., 1998) Reactive method is an autonomous control of an entity to overcome disturbances by itself, while the collaborative method is used for a cooperation of an entity with other entities in order to adapt to disturbances. To implement reactive/collaborative methods, the distributed control architecture is required (Park \& Lee, 2000; Park \& Tran, 2009). The control architecture changes from centralized control of nonintelligent entities in hierarchical structures of the FMSs towards decentralized control of intelligent entities in distributed structures. The new trend of the manufacturing system development is to apply autonomous behaviours inspired from biology into the manufacturing systems. The biological organisms have two types of information: genetic information (DNA-type) and knowledge information (Brain/Neural-type or BN-type). The DNA-type information evolves through progressive generations while the $\mathrm{BN}$-type information is achieved during the lifetime of one organism by learning (Brussel, 1995). Unification of information makes organisms to show functions such as self-recognition, self-growth, selfrecovery and evolution (Ueda et al., 2000). In the genetic manufacturing system (GMS), the information classification of manufacturing systems based on the information types of biological organisms was proposed. The DNA-type information includes the data of products and manufacturing equipments. The data of products determine which processes are required by the product while the data of manufacturing equipments show which machines the corresponding processes are operated. On the other hand, the BN-type information consists of the rules for cooperating machines in order to carry out processes. The technologies for embedding this biological information to the machines and work-pieces such as RFID, magnetic magnesium were proposed. The GMS concept was improved by the recent researches in which the intelligent behaviours of the system adapted to biological organisms were added. The concept of biological manufacturing system (BMS) is proposed by Ueda et al. (Ueda et al., 2006) as shown in Fig.16. In the BMS, machine tools, transporters, robots, and so on should be seen as biological organisms, which are capable of adapting themselves to environmental changes. In order to realize BMS, agent technology was proposed for carrying out the intelligent behaviours of the system such as self-organization, evolution and learning. The reinforcement learning method was applied for generating the appropriate rules that determine the intelligent behaviours of machines. However, this system only achieves a greater efficiency if the agents are equipped with the cognitive capabilities, which improve 
the autonomous behaviours of the agents. Currently, the cognitive technology which equips the cognitive behaviours for the system such as perception, reasoning, and action is applied independently with the agent technology in the manufacturing applications.

In the holonic manufacturing system (HMS), the ADACOR holonic manufacturing control architecture was proposed as shown in Fig.17 (Leitao, 2008). In this architecture, the manufacturing control architecture is divided into holons (Christo \& Cardeira, 2007) such as the product (ProdH), task (TH), operational $(\mathrm{OpH})$, and supervisor holon $(\mathrm{SupH})$. Operational holons $(\mathrm{OpH})$ represent the physical resources available on the shop floor.

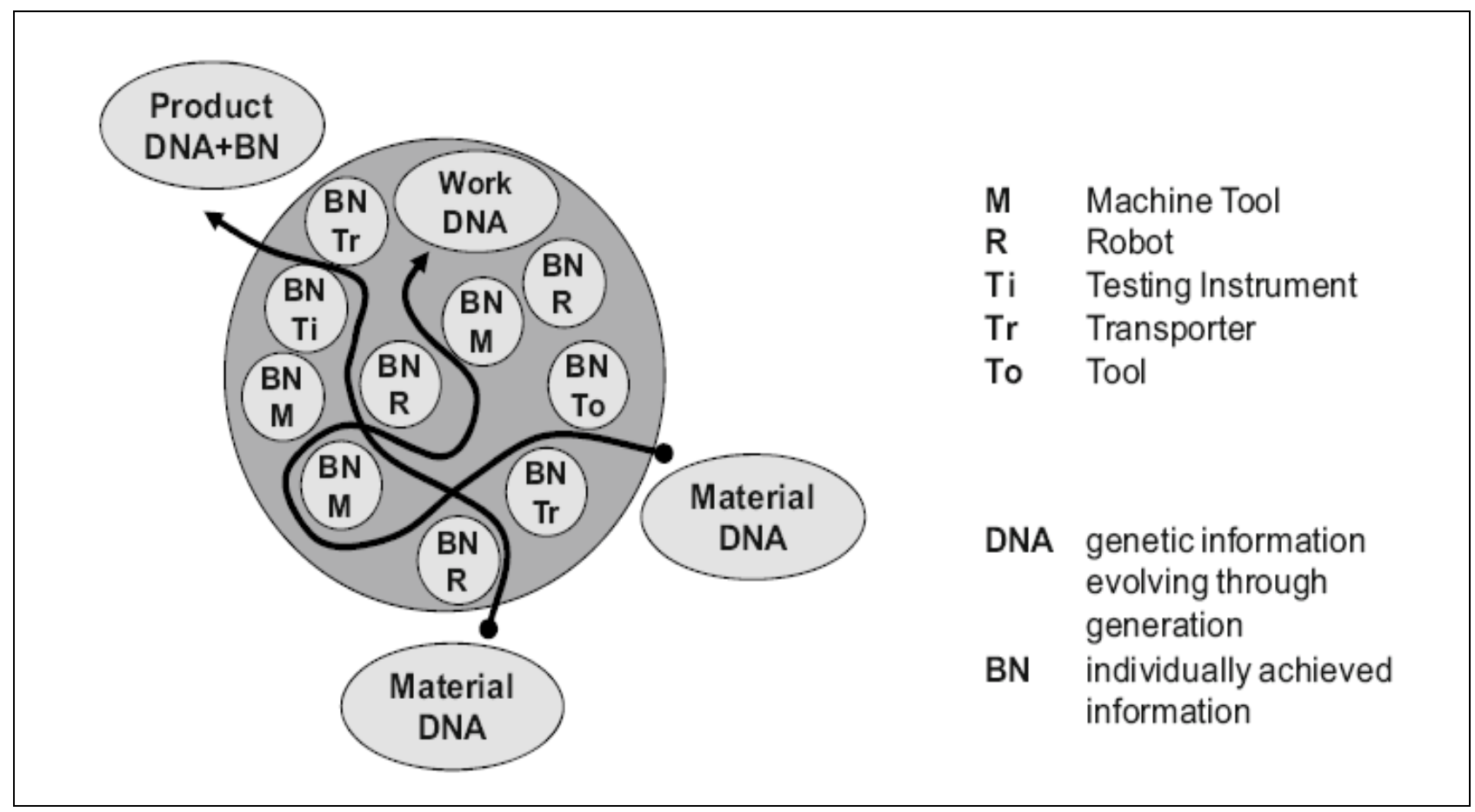

Fig. 16. Concept of a biological manufacturing system (Ueda et al., 2006)

These holons adapt to unexpected disturbances such as the machine breakdown, tool-wear and so on by themselves or by the interaction with other operational holons through a supervisor holon ( $\mathrm{SupH})$. The mechanism for adapting to disturbances is based on pheromone techniques inspired from the ant colony optimization. The pheromone parameter indicates the level of impact of the disturbance. In this architecture, there still exists the weakness of traditional centralized and sequential manufacturing systems due to the use of the supervisor holon that reduces the flexibility of the system to respond to disturbances. This weakness will be overcome by a decentralized control architecture in which the agent technology is applied for implementing the logical part of operational holons so that these holons can directly interact among them for overcoming disturbances. 


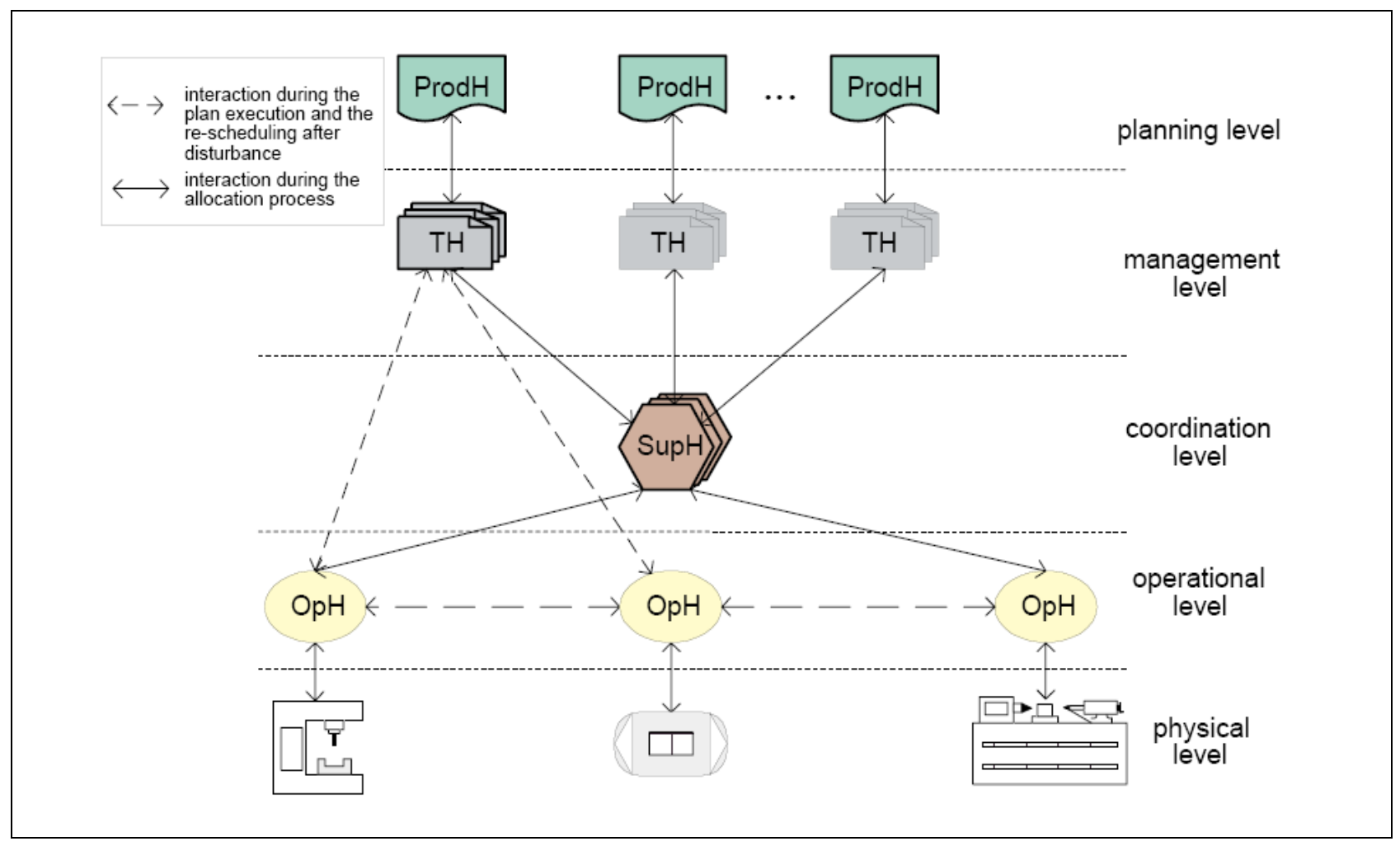

Fig. 17. Concept of an ADACOR holonic manufacturing system (Leitao, 2008; Leitao, 2004)

Autonomy in manufacturing systems is shown by the self-learning capability. Self-learning is one of the advanced characteristics of the modern manufacturing systems. This characteristic allows the system to improve the current capability, to diagnose the status. The cognitive models have been studied to apply into manufacturing systems to equip the system with the learning capability. The machines are equipped with the artificial cognitive capabilities so that they can communicate, cooperate, and negotiate to get the optimal goal of the manufacturing process.

\section{Self-optimizing Processes}

The evolution of control techniques toward the intelligent machine for future is shown in Fig.18. The first innovation focuses on developing the hardware for machine tools that brings the high speed, high precision, and high productivity. In the second innovation, the software for generating automatic operations and adaptive control are focused. The machines can carry out multiple tasks, and multiple functions. The third innovation focuses on the autonomous operations with applying of artificial intelligence. In this innovation, new concepts of machine tools have been proposed such as reconfigurable machine, self-maintenance machine, and ubiquitous machines. 


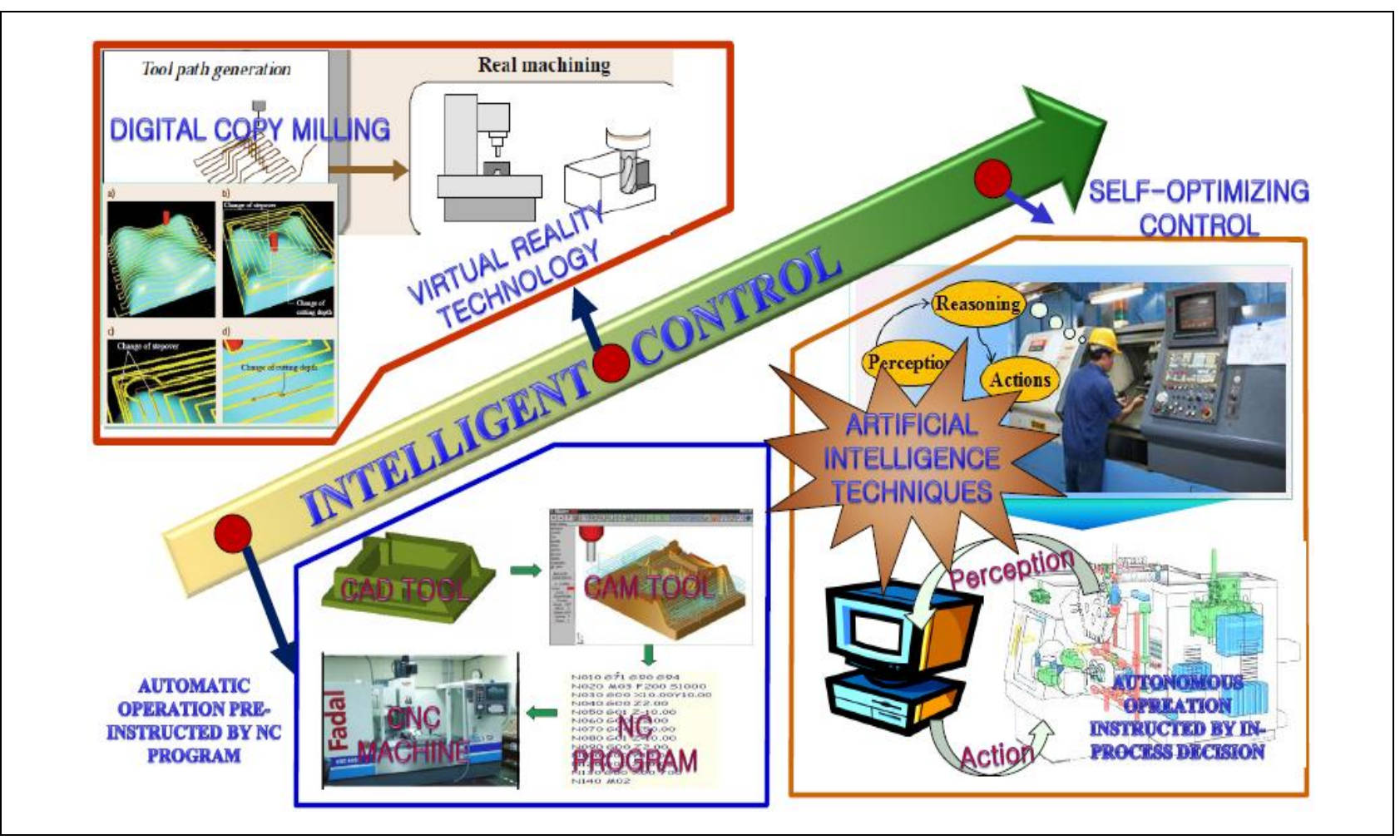

Fig. 18. Trend of intelligent control techniques

\subsection{Concept of Self-optimizing Process}

In the machining system, the machine tools play an important role to have a higher value added products with low production costs and high productivity. Their performance has a high effect on both the performance and productivity of the machining system. However, the conventional Computer Numerical Control (CNC) system cannot allow achieving an in-process quality control due to its control architecture. The current control architecture has two major levels which are the servo control (level 1) and the interpolator (level 2) for the axial motion control of machine tools as shown in Fig. 19. The current CNC system, which is only suitable for the static product planning, enables to achieve highly sophisticated motion control, but it cannot enable to achieve sophisticated cutting process control. In the case of disturbances such as tool wear, the initial optimal values of cutting parameters must be changed to ensure the product quality which never happens in the conventional CNC system. In the conventional machine tools, the machining sequence or cutting conditions is not generally allowed to change during machining operation. The conventional CNC system allows performing only automatic machining operations pre-instructed by NC programs that are the cause to the reduction of machining operation's flexibility.

To adapt to disturbances in consideration of the product quality, this article presents a self-optimizing control system. The proposed control architecture is shown in Fig.19. For a self-optimizing control system to achieve more sophisticated process control, two additional levels of control hierarchy, levels 3 and 4, are required. To enable an intelligent process monitoring, the self-optimizing control scheme is assigned at a higher level (level 3) of the control hierarchy which can detect 
machining state independently of cutting conditions and machining operation. The supervisory level is level 4 which receives feedback from measurements of the finished part. This level enables to use effectively the feedback information regarding the cutting results to improve the next machining processes. For realizing this level, a reasonable index to evaluate the cutting results and a reasonable strategy to improve cutting results are required. Additionally, an autonomous process planning strategy generating a flexible and adaptive working plan is required as a function of the new $\mathrm{CNC}$ system which must be responsive and adaptive to unpredictable changes of the machining system. For this purpose, the utilization of knowledge, knowhow, and skill related to machining operations has to be considered. To realize a dynamic adaptation to the process, self-optimization can be characterized by three joint actions:

- Analyzing the current situation: the machining system determines its current situation by analyzing setting parameters and signals from sensors that detect the state of the system with respect to the objectives.

- Determining objectives: Optimization requires the determination of internal objectives that define how the machining system shall perform in order to achieve external objectives. The internal objectives parameterize the subsystems and actuators.

- Adjusting system behaviour: Comparing the current situation with the system objectives yields the optimization potential. This enables the machining system to decide about the adaption of internal objectives in order to achieve the external objectives.

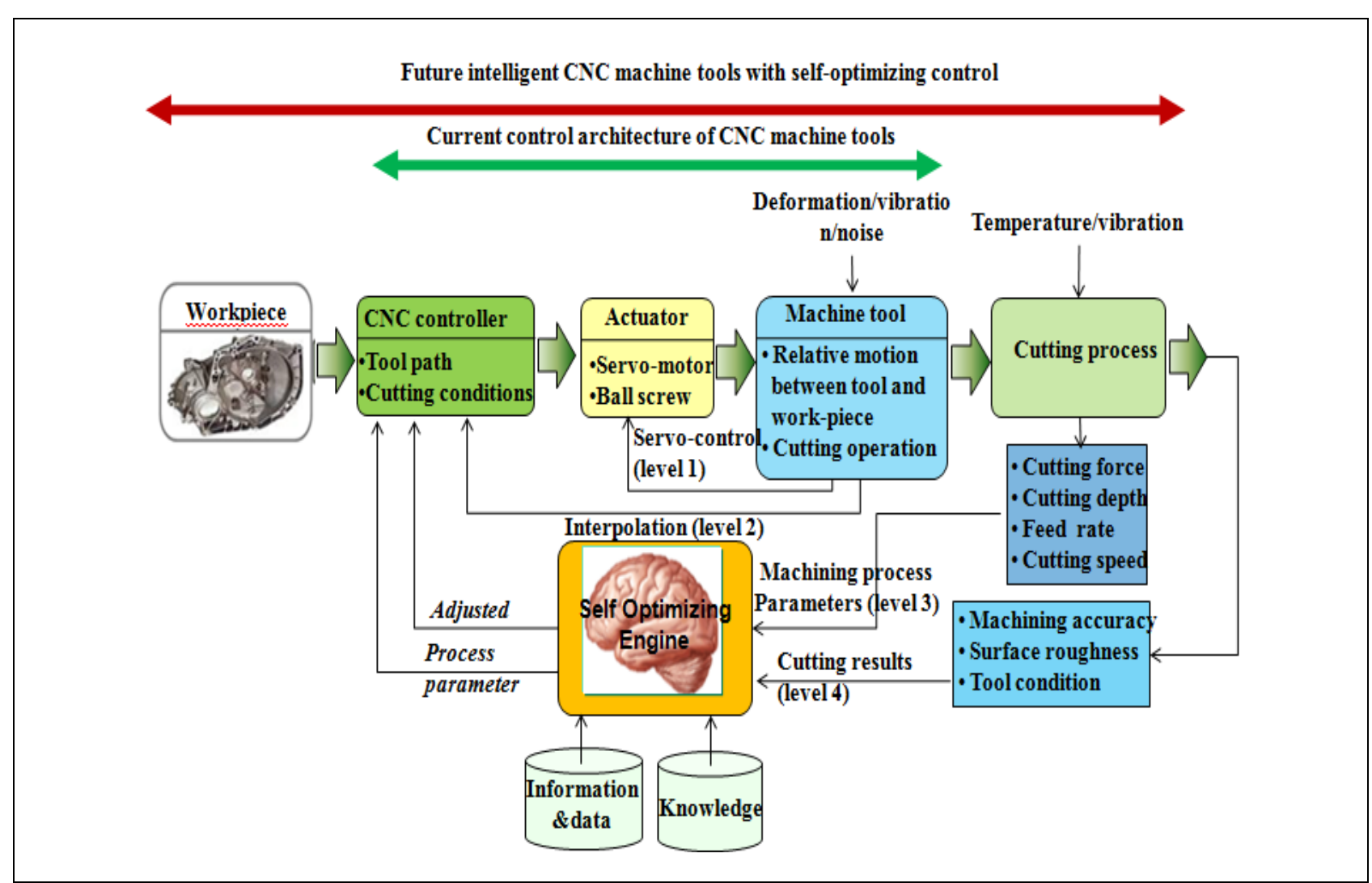

Fig. 19. Concept of self-optimizing control system 


\subsection{Technologies for Realizing the Process Self-optimization}

The proposed self-optimizing control system involves monitoring and selfadjusting the cutting conditions using the information convergence technologies as shown in Fig.20. The monitoring includes the act of identifying the characteristic changes of the machining process based on the evaluation of process signatures without interrupting normal operations. Basically, a monitoring process has two parts: sensing and monitoring. In sensing, appropriate signals used for tool wear monitoring are obtained from sensors. In monitoring, composed of signal processing and decision making can be divided into model-based and feature-based methods. Both methods use sensor signals from the cutting process for the system input. For realizing the monitoring system, the core technologies must have as follows:

- Multi-sensor system: using more than one sensor for monitoring machining processes and tool conditions.

- Automated feature extracting system: enabling to automatically generate the monitoring features. The signals sensed from multiple sensors are analyzed, compacted, and selected by the system to generate the most sensitive features to the monitoring subjects. The extracted features are also further refined or reselected by the monitoring system.
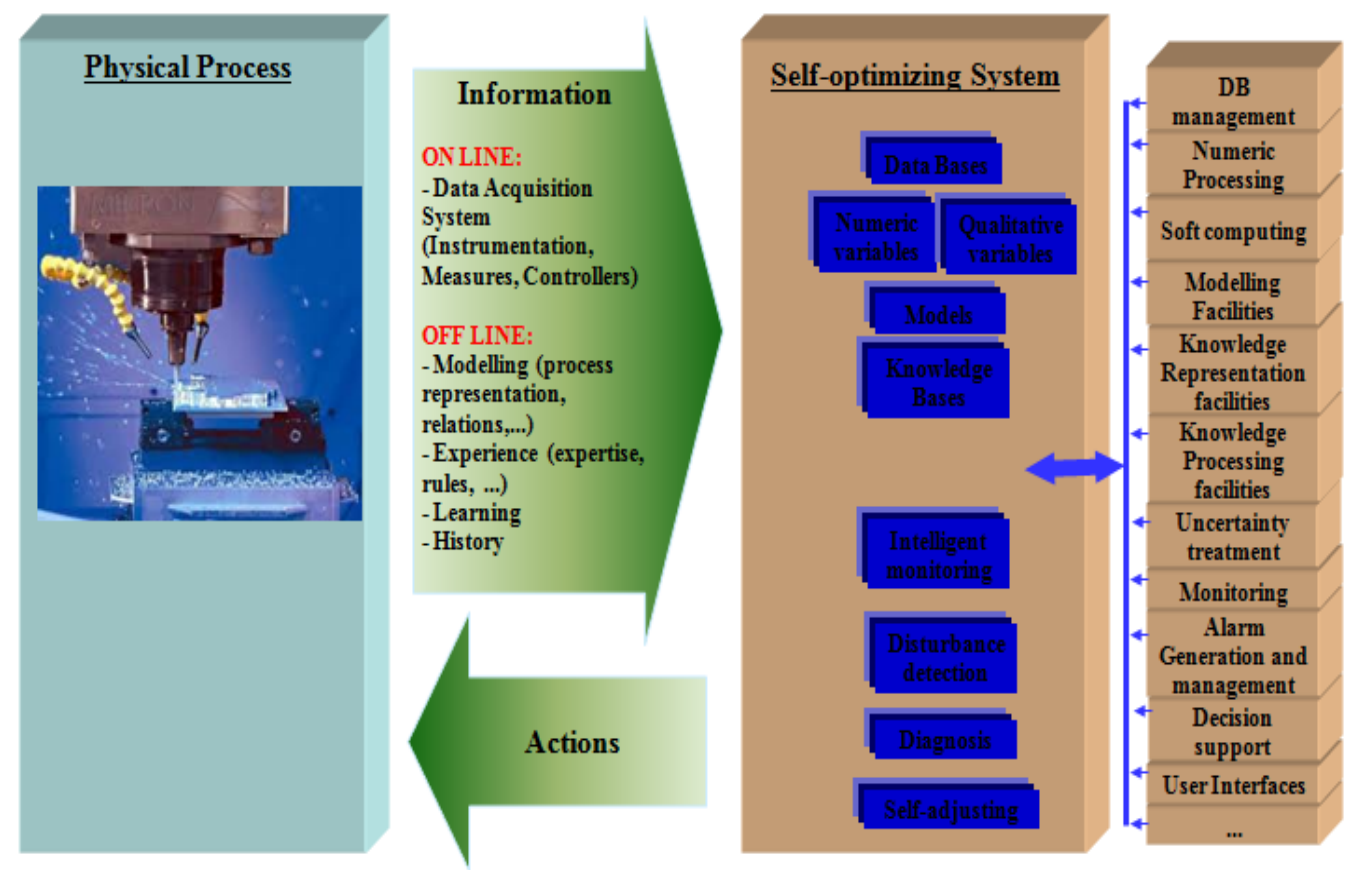

Fig. 20. Functional architecture for a self-optimizing machining system

For self-adjusting the cutting condition according to the results from monitoring, artificial neural network technique are applied. The main advantages in using neural networks over other methods are easier inclusion of new input parameters, and automatic learning of non-linearity in the tool wear process. The self-adjusting cutting condition concerns on the machining quality criteria as well as the economical 
criteria. In the machining quality, the cutting parameters, tool wear and so on are considered to achieve surface quality, and accuracy of geometrical profile. In the economical criteria, optimizing the cutting parameters is to maximize the objective functions that relate to economic goals such as production cost, production rate.

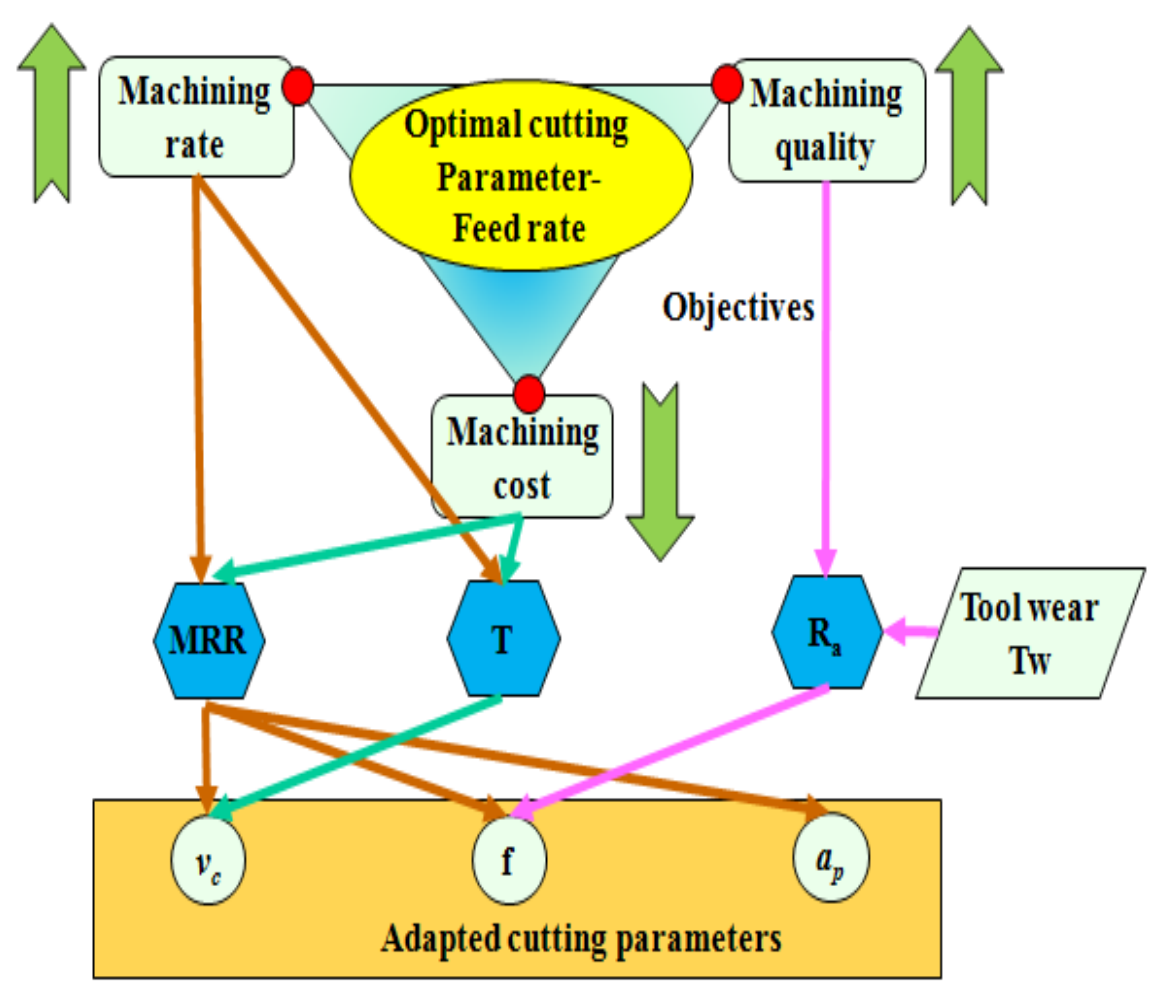

Fig. 21. Objectives of an optimal machining process

The objective function is designed according to the relationship between process parameters and quality factors as well as the mathematical model of the relation between the force, tool wear and product quality in machining process. The main objective of machining operations on the machine tool is to machine products with low cost and high quality. In order to achieve this goal, the determination of optimal machining parameters such as cutting speed (vc), feed rate (f), and depth of cut (ap) plays an important role as shown in Fig.21. The generating optimal cutting parameters ( $\mathrm{vc}, \mathrm{f}, \mathrm{ap})$ is considered in relation of the objective functions such as cutting quality (represented by the surface quality $\mathrm{Ra}$ ), production rate, and operation cost. The production rate and operation cost are determined via the functions of the metal remove rate (MRR) and tool life (T).

\subsection{Working Behavior within Self-optimizing Process}

Fig. 22. shows the self-optimizing and self-adjusting mechanism during machining process for adapting to the tool wear disturbance. The MES system dispatches the process plan of each machine according to the identification information of machine tools (which are called machine tool ID numbers). The green light is $\mathrm{ON}$ in the case of the normal state of a machining process. The amount of tool 
wear is predicted using neural network technique. Then, the cutting parameters are adjusted by the calculated values for compensation of the current tool wear in real time to keep the consistent quality of the machined product.

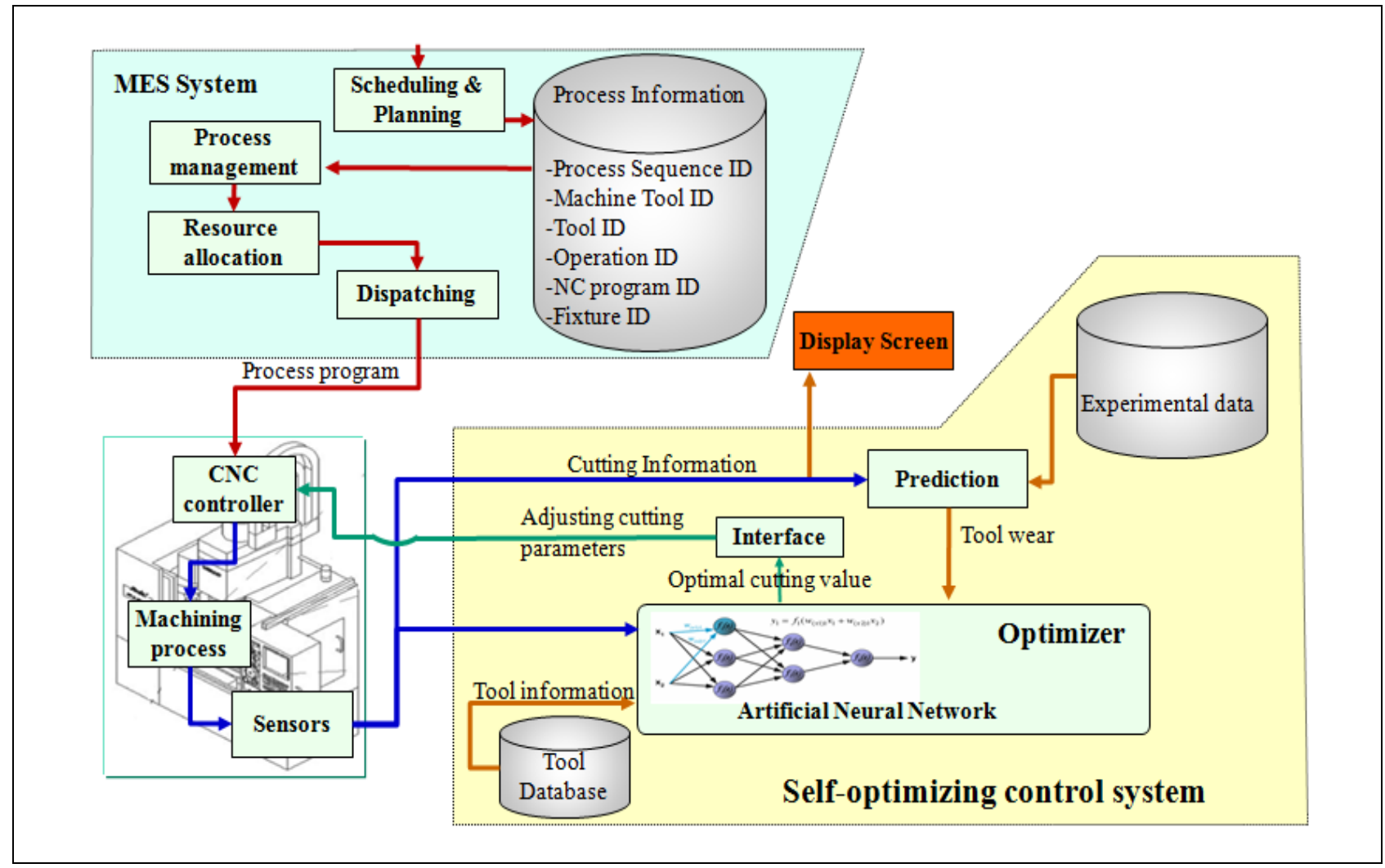

Fig. 22. Optimal cutting parameters in the case of tool wear

\subsection{Implementation of Self-optimizing Process}

The machining shop considering as the research object is consisted in 12 machines for 17 processing operationsas shown in Fig.23. There were 685 disturbances happened during three years. Due to the manual recovery, the system utilization was too low. Applying the self-optimizing control to the conventional machining system was tested successfully. The self-optimizing milling process at the machine center \#3 enables to keep the quality of the machined product in consideration of the tool wear. During machining process, the amount of current tool wear is predicted using Artificial Neural Network (ANN). The neural network configuration is 6-10-1. The network is consists of 6 input neurons corresponding to 6 input parameters such as cutting force, feed rate, depth of cut, processing time, cutting speed, and initial tool wear. There is one hidden layer with 10 neurons. The off-line training was stopped after 2000 training iterations and the minimum error achieved was 0.025 . The formula for calculating the surface roughness of the machined part in consideration of the predicted tool wear is given as follows (Pal et al., 2011). The Ra must in the range of the allowed limit.

$$
R_{a}=\frac{0,125 \times f^{2}}{r_{E}} \times\left(1+1,6103 \times T_{w}\right)^{0,7315}
$$


where $\mathrm{rE}$ is tool nose radius $(\mathrm{mm})$; $\mathrm{f}$ is feed rate $(\mathrm{mm} / \mathrm{rev})$; and Twis amount of tool wear (mm). To keep the consistent quality of the machined product, the new cutting parameters are determined using another artificial neural network with the depth of cut, cutting speed, and the amount of tool wear at the input. The output is the optimal feed rate. The neural network configuration is 3-9-9-1. There are two hidden layers with 9 neurons for each layer. The off-line training was stopped after 2000 training iterations and the minimum error achieved was 0.28. Two ANN models were tested showing the reasonable results in comparison with the experimental data.

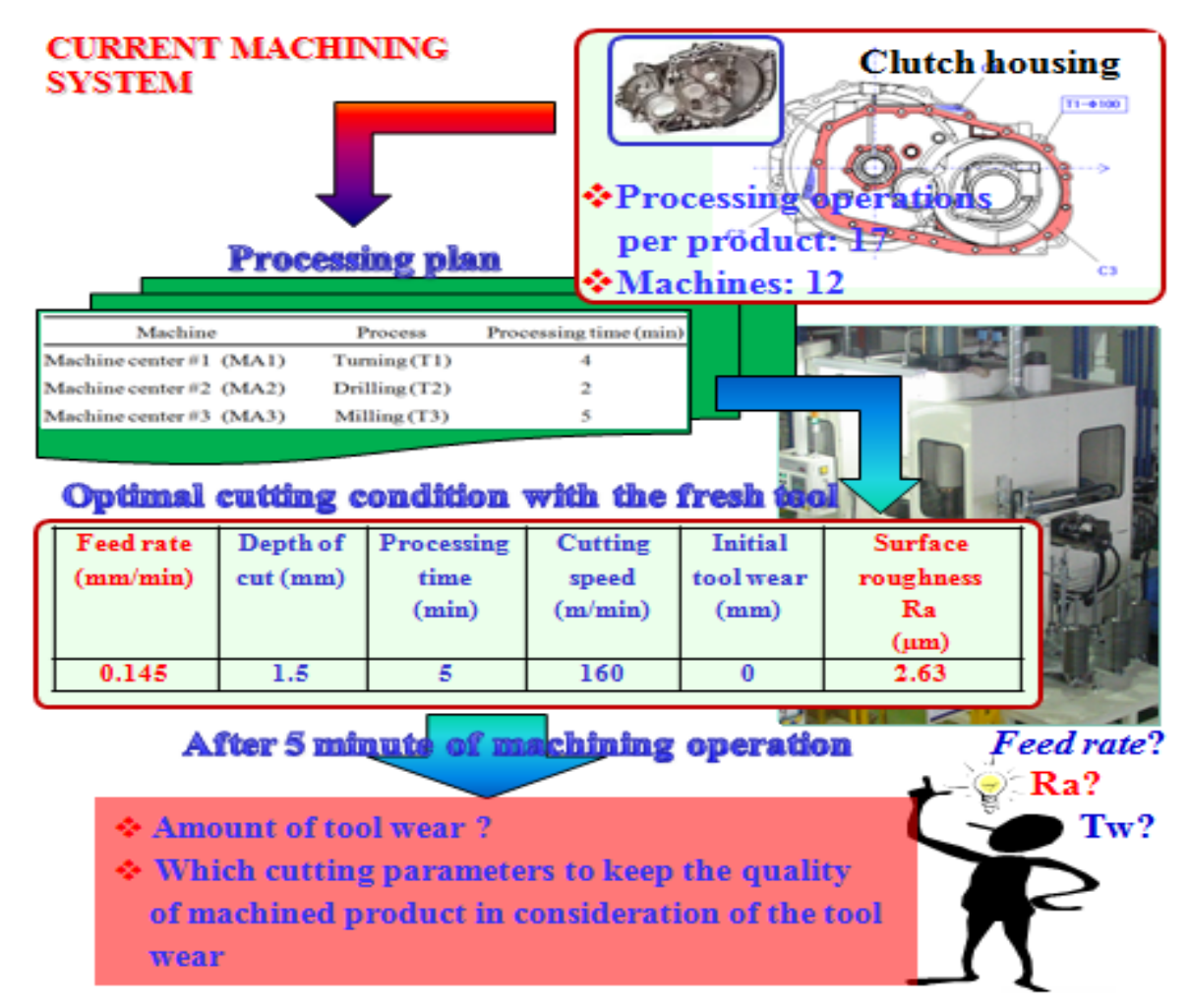

Fig. 23. Conventional machining system

\section{Autonomous Manufacturing System}

\subsection{Concept of an Autonomous Manufacturing System}

The architecture of a machining system based on cognitive agents for adapting to disturbances is shown in Fig.24. A multi-agent system was developed to keep the manufacturing process running when disturbances appear. In this application, resources of the machining shop are controlled by cognitive agents in the case of disturbances; otherwise, the MES controls the shop floor. Cognitive agents are divided into functional agents including work-piece agent, transporter agent, machine agent, and robot agent. This division is based on functions which agents undertake in the machining shop. Work-piece agent manages the processing state of a work-piece. Each work-piece is assigned by an identification number stored in the RFID tag that was attached on the work-piece. Every machine on the shop floor is represented by a 
machine agent. This machine agent has knowledge about its machine's physical, process capabilities, probable tooling and schedule. The machine agent receives the sensor information from the machine about the machine status and processes. This enables the machine agent to inform the work-piece agent when the processing starts and in particular, when the processing ends and the work-piece leaves the machine. The transporter agent contains routings of the transporter, consequently, interacting with the work-piece agent to transfer the work-piece to the corresponding machine tool for processes. The robot agent contains the information of the robot, about the operations, availability, and interactions with the machine agent to put or take the work-piece to the machine tool.

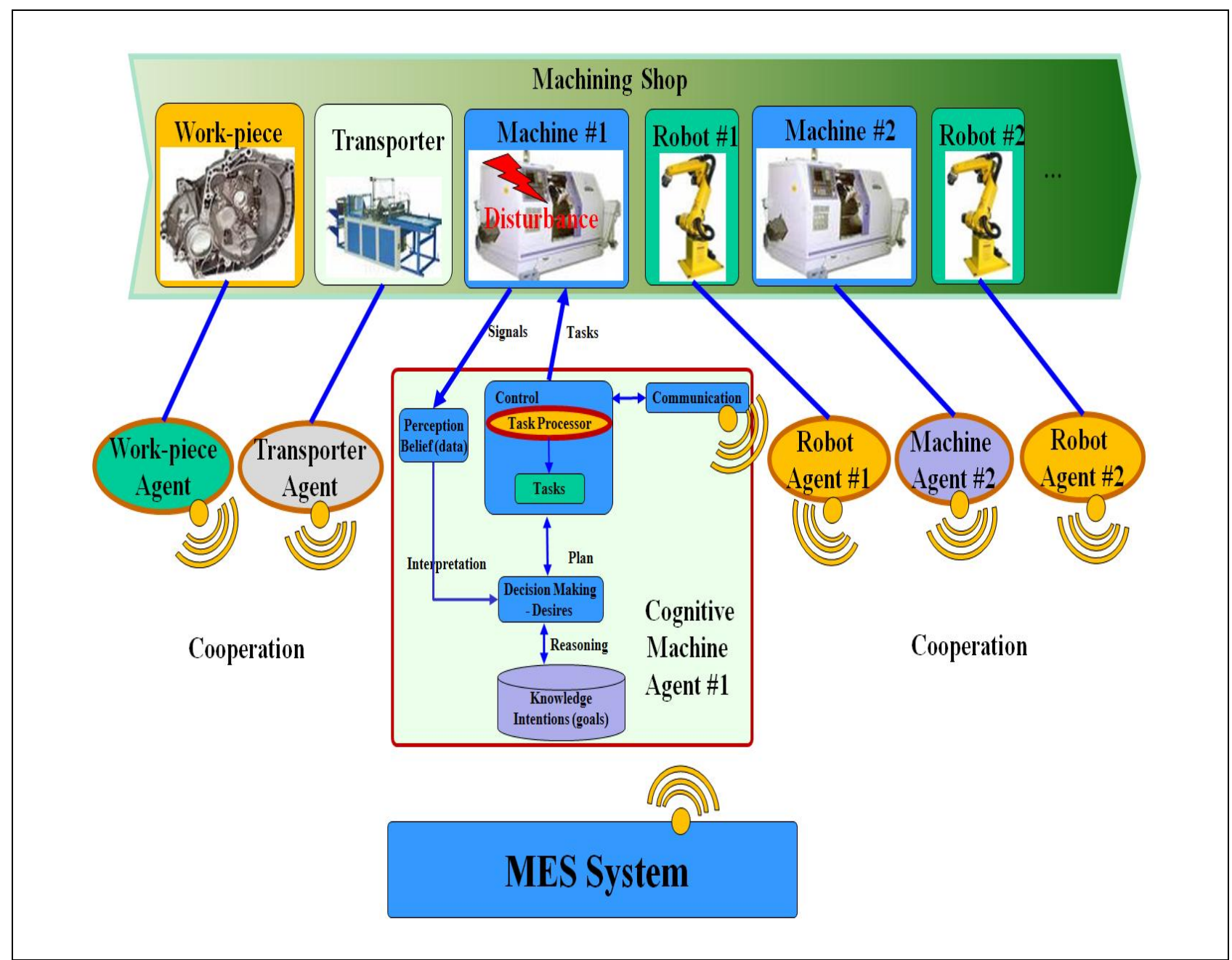

Fig. 24. Model of a machining shop based on agents.

\subsection{Technologies for realizing an AMS}

In order to accomplish the activities of an IMS-CA, the information and their flow were modelled as shown in Fig.25. The MES information for scheduling and planning, dispatching, and process management, is supported to generate the process and operation plan as well as to monitor the process and schedule.

The information of the machine agent includes the information of its own machine's physical and process capabilities, as well as the information for reasoning 
and decision making. This information is used for carrying out the composed machining process and the cooperation with other agents. The information of workpiece, transporter, and robot agent delivers their own data to support the decisions of other agents and communicate for deciding where to go.

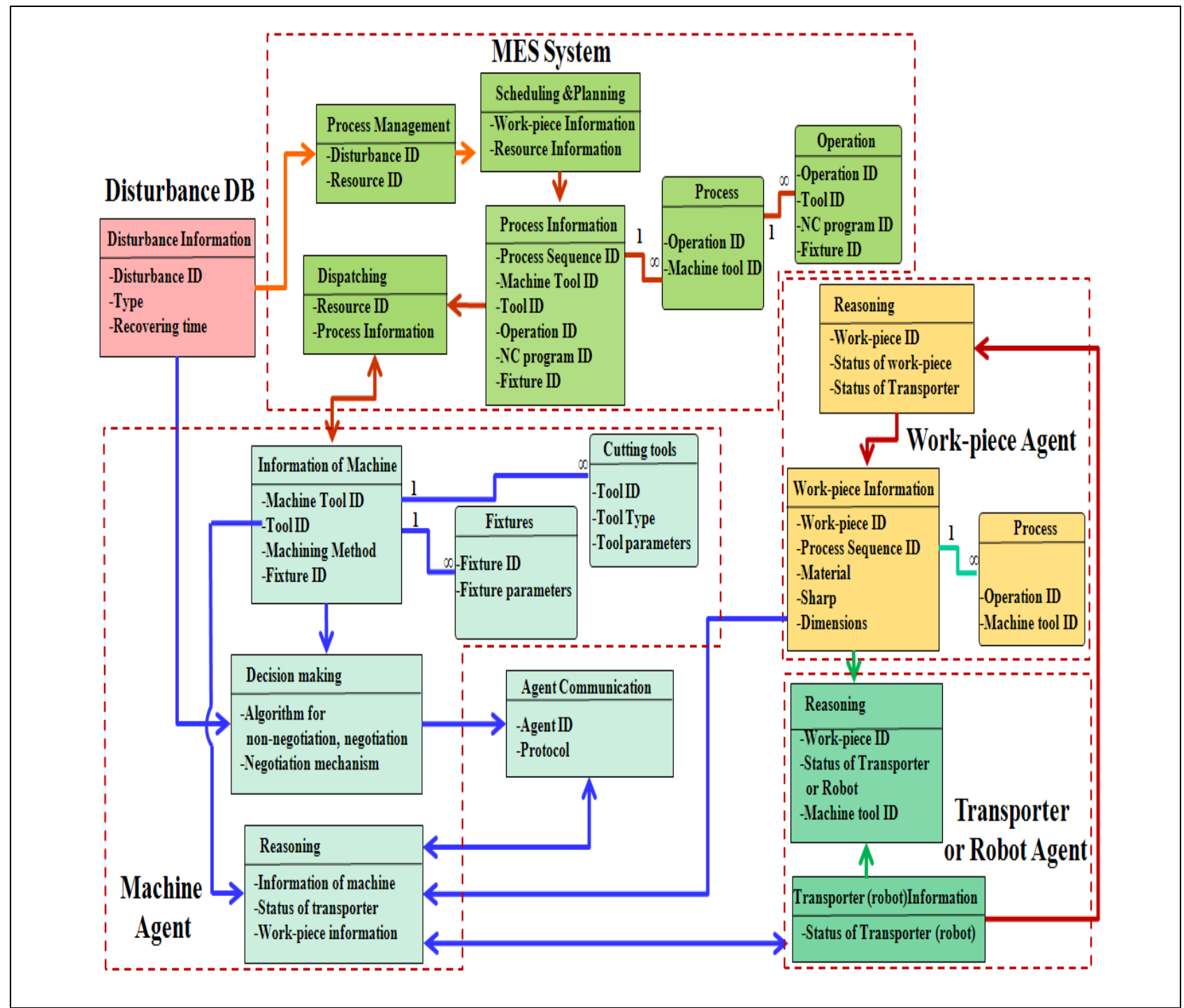

Fig. 25. Information model of an autonomous machining system

The reasoning process of human, namely practical reasoning mechanism was applied to the agent technology to implement the cognitive agent for adapting to the disturbances as shown in Fig.26. From this reasoning mechanism, the disturbance is recognized through a comparison between the current state of the machining task (denoted by Beliefs (B) and Intentions (I)) and the Desires (D) of the processed task. The disturbance is classified according to the criteria presented in Section 1. If the disturbance belongs to the non-negotiation type (type B), the agent overcomes the disturbance with its own knowledge. In case the solution cannot be found or the disturbance belongs to the negotiation type (type C), the negotiation process is carried out. The agent that can execute the given task is selected through the agent 
negotiation within the machining shop and carries out that task. If all agents cannot execute the task or the disturbance belongs to the rescheduling type (type A), a rescheduling is done by the Manufacturing Execution System (MES).

When the disturbance which belongs to the negotiation group happens to the machine during carrying out the operation dispatched by the MES, we need an alternative machine to carry out that operation in order to keep the given schedule within the tolerance range. So, we consider only the disturbed operation at that time occurring the disturbance, not all operations for machining the clutch housing. Due to using the machining center, there are several machines in the machining system which can carry out this operation.

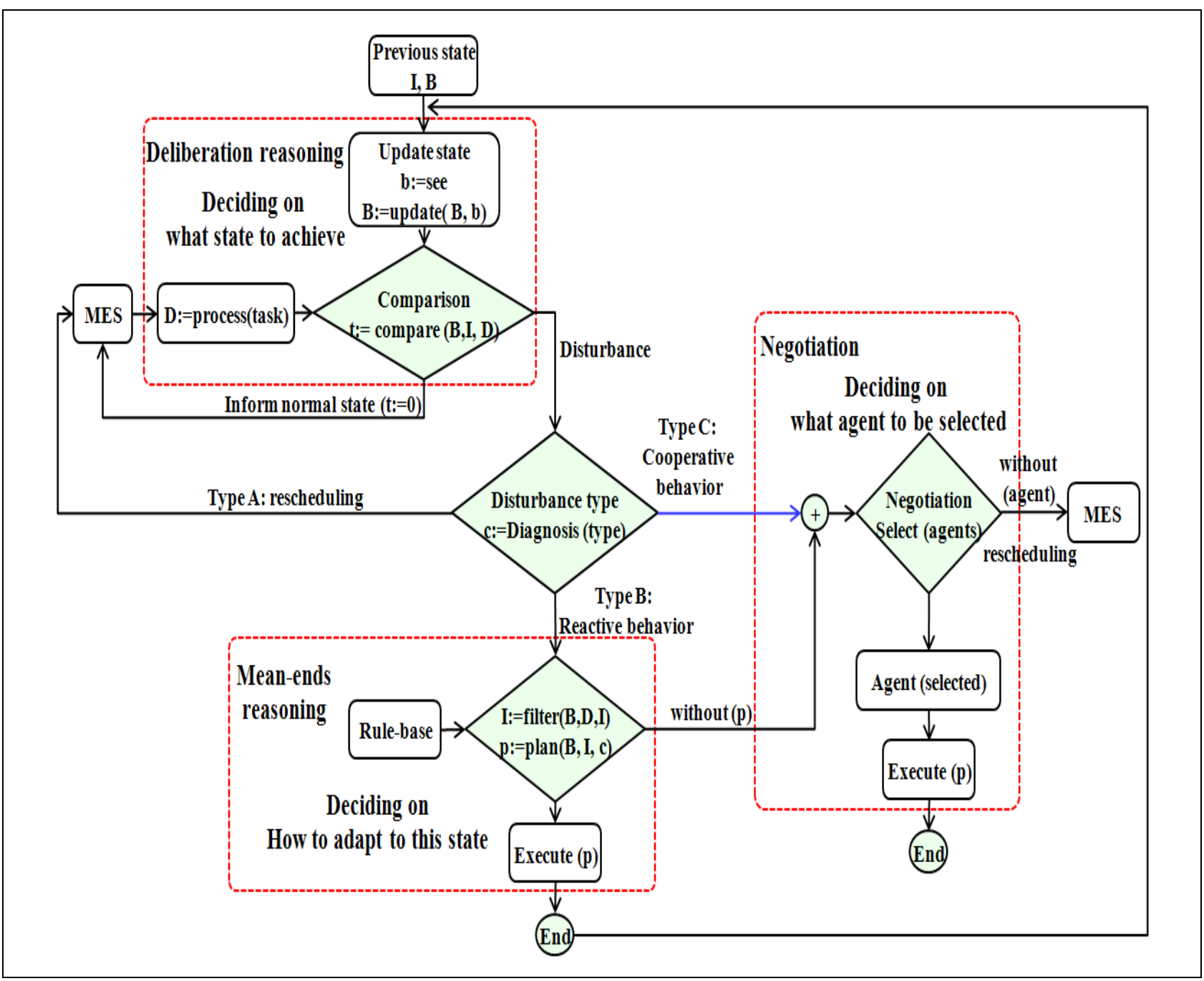

Fig. 26. Practical reasoning mechanism of a cognitive agent

Therefore, we must choose a most appropriate machine among the alternative machines.To select the most appropriate machine, the machine agent \#1 managing the failure machine sends the task information to the remaining machine agents. The task information consists of the machining method, the cutting conditions, and the tool type. The machine agents compare these information to their machine ability through their database. In the database, potential factors of a machine for carrying out 
a task such as machine specification and capability to machine work-piece according to its functional requirements are stored. Each machine agent is considered as an ant, and the pheromone is used as a communication mediator in agent negotiation. The function of pheromone is to indicate the ability of machine for carrying out the task roughly. In agent negotiation, pheromone value is used as the criterion for choosing the optimal machine among the alternative machines. In case the machine agents meet the requirements of the task, they generate the pheromone values. Otherwise, the pheromone value equals zero.Based on the ant colony algorithm (Xiang \& Lee, 2008), the formulation for calculating the pheromone value was designed in consideration of the executing ability, processing time and machining cost. It is shown as follows:

$$
p_{M A_{i}}=\frac{q}{\alpha_{t} \times \frac{M_{t}}{M_{t o}}+\alpha_{c} \times \frac{M_{c}}{M_{c o}}}
$$

$\mathrm{q}$ is the executing ability of the machine MAi about the task asked from the failure machine. In the executing ability, the functional requirements of the work-piece such as dimension, tolerance, surface roughness and micro structural change must be fulfilled. If the task $\mathrm{t}$ can be carried out at the machine MAi, $\mathrm{q}=1$, otherwise, $\mathrm{q}=0$. $\mathrm{Mt}(\mathrm{min})$ and $\mathrm{Mc}(\$)$ represent the processing time and machining cost of the task $\mathrm{t}$ at the machine MAi, respectively. Mtoand Mco known by the originally planned machine are the minimum machining time and machining cost of the task t,respectively. The highest pheromone value of the task requires the lowest processing time $(\mathrm{Mt})$ and machining cost $(\mathrm{Mc})$.

The factors $\alpha \mathrm{t}$ and $\alpha \mathrm{c}$ represent the weight of the machining time and cost, respectively. The weight of these two influences is different under the different circumstances of the market. For example, in an economic boom, the machining time is critical; in a depression, the machining cost is more important.

\subsection{Working Behavior within AMS}

Fig. 27 illustrates the mechanism of cognitive agents to adapt to the disturbance happened at the machine tool. At the beginning, the MES sends a task command to both of the controllers and cognitive agent (denoted by 1). The cognitive processor identifies the goals and transforms them into the desires. The machining shop state is updated by the monitoring module. This module then filters the data to obtain the information corresponding to the responsibilities of the agent. In case disturbances occur, the data are categorized into high and low frequencies through a feature extraction unit. Depending on the types of high or low frequency signal, fuzzy logic is used for diagnosis with low frequencies, and neural network is used for high frequencies. Diagnosis results report the machining shop states: disturbed or normal 
status (denoted by 2). The planner compares the data from the output of the diagnosis module with the desired goals. If the data reach the desired goals, a message is sent to the MES to report the normal state of the machine (denoted by 3), and the shop floor continues running. Otherwise, the machine agent makes a decision based on the disturbance classification. If the disturbance belongs to non-negotiation type, the decision maker generates a new plan based on the data, desires and intentions (denoted by 4). This plan is directly done by the machine in which the disturbance happens (denoted by 5). This case is illustrated by the disturbance such as tool wear where the machine agent adjusts cutting conditions without affecting to the quality of product.

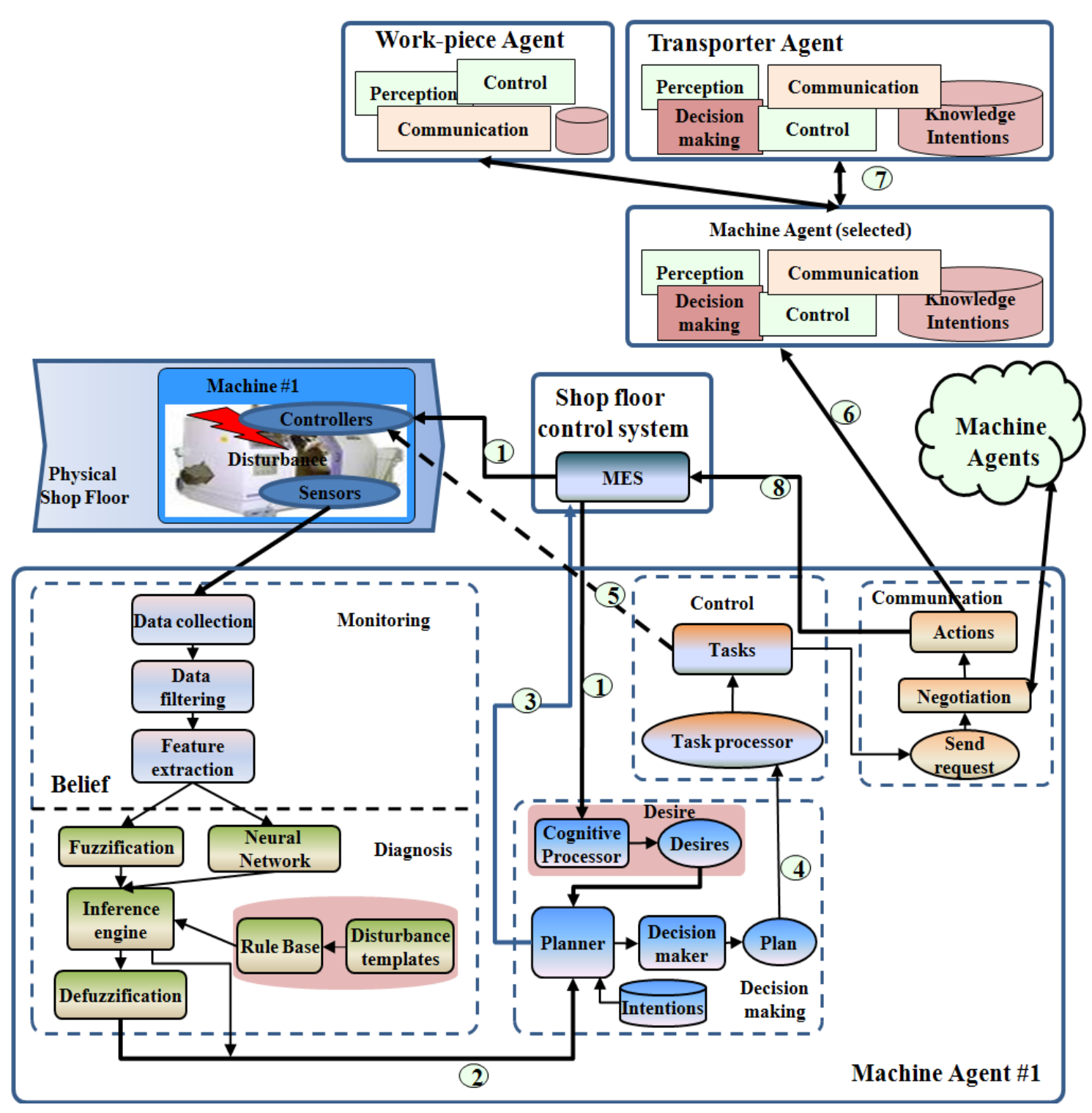

Fig. 27. Mechanism of cognitive agents for adapting to disturbances

In case the disturbance belongs to the negotiation such as the machine breakdown, this plan is done by another machine. The cognitive agent implements a negotiation with other agents as shown in Fig.28. It sends a request for help to all machine agents. The best solution is chosen based on the evaluation of alternative 
machine agents in case many machine agents satisfy the requirements. The negotiation mechanism is based on the ant colony technique. The job of the disturbed machine is then performed by another machine in order to keep the manufacturing system running (denoted by 6 ). The selected agent sends a message to the work-piece agent and transporter agent (denoted by 7) to report that it performs the work of the machine in which the disturbance happens. The machining system uses the previous plan when the disturbed machine is restored.

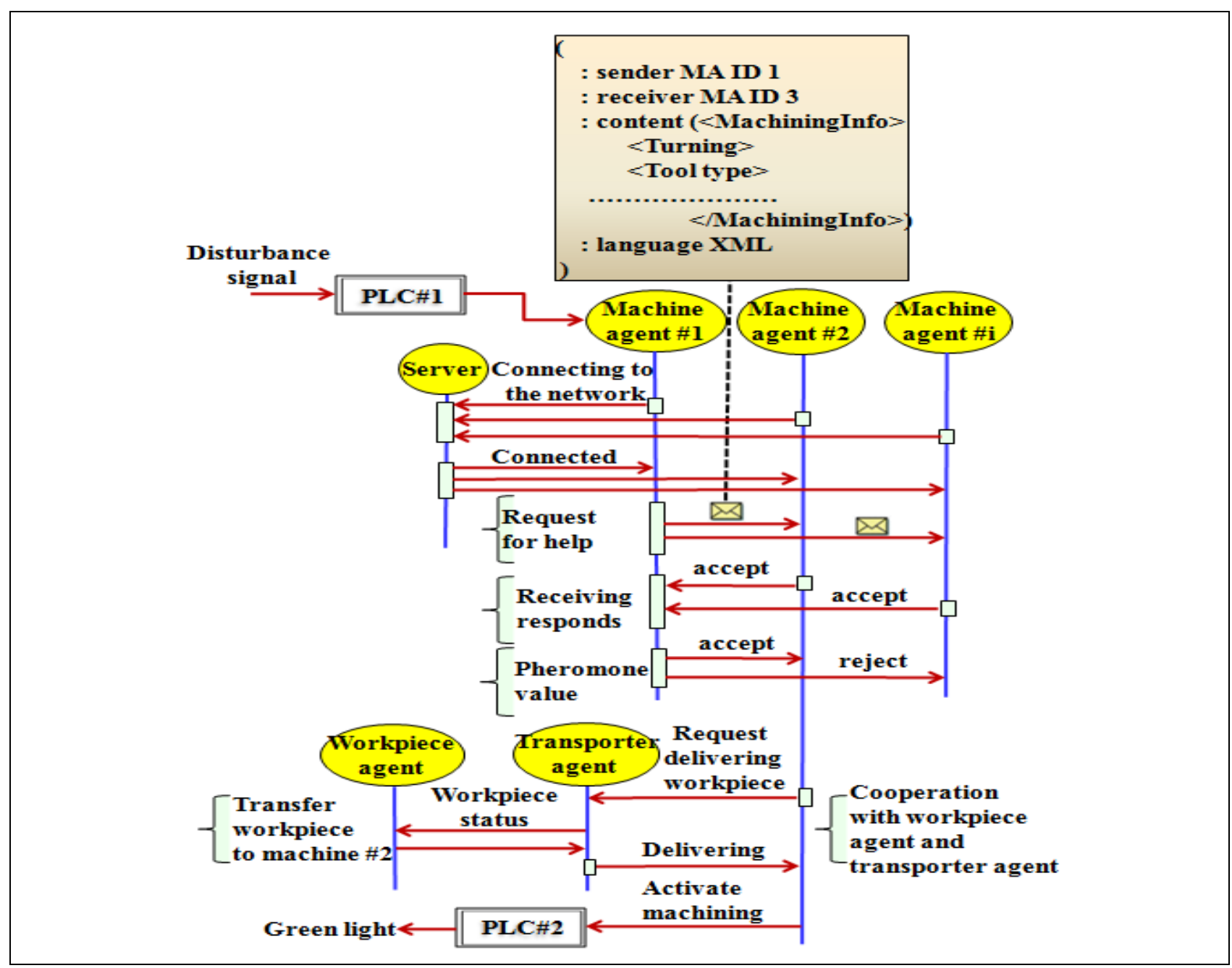

Fig. 28. Generating the best route of workpiece in the machining system

This solution is applied to disturbances, which take a short time for recovery. In case the disturbances cause a long recovering time or the negotiation among agents does not have any solution, the request is sent to the MES for rescheduling (denoted by 8). If disturbances occur with the robot and transporter such as the malfunction of controllers, the robot agent or transporter agent depends on the disturbed level to use a non-negotiable plan or a negotiable plan or request rescheduling for overcoming disturbances.

\subsection{Implementation of AMS}


The cognitive agents were developed using the .NET platform and C\#. The system architecture of the cognitive agent-based machining shop is shown in Fig.29. It points out the three kernel issues to implement the cognitive agents, which are the interaction protocol, agent behaviors, and database (DB) as well as the information flow among components in the system for carrying out the functionality of the proposed AMS-SCA. The agent interacts with the MES and the other agents via the XML messages. The process control protocol (OPC) for linking and embedding objects is used for communicating the agent with PLC which connect to the physical devices on the machining shop such as sensors, disturbance input, and alarm device.

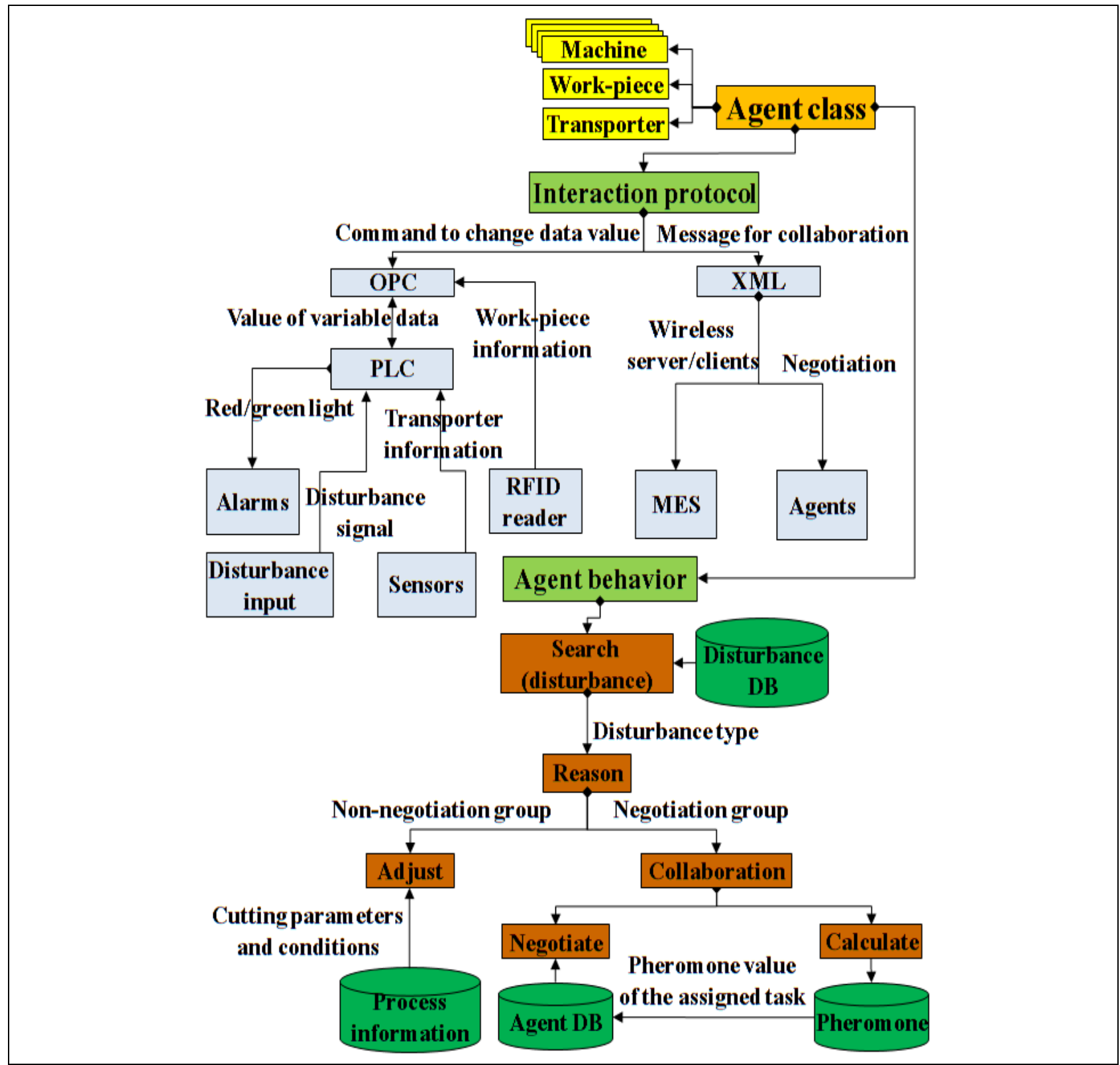

Fig. 29. System architecture of the cognitive agent based machining shop

The databases, including the process information, the agent addresses for communicating in the network, the pheromone values of the tasks related to the machine agents, and the disturbance DB, were built using SQL ServerTM 2005. The agent uses the "search" method to diagnose and classify the disturbance. According 
to the disturbance type, the agent reasons to make a decision using the "adjust" or "collaboration" methods. In collaboration, the agents generate the pheromone value of the assigned task using the "calculate" method. Then, the "negotiate" process is carried out among agents to find the agent with the highest pheromone value for carrying out the task.

The screen shot of the developed system in the case of the tool broken is shown in Fig.30. Self-optimization at system level is shown by the generating the optimal route for workpiece in case one machine in the machining system breaks down. The process control protocol (OPC) for linking and embedding objects is used for communicating the agent with the CNC controller. The optimal route is generated using the ant colony technique for cooperating agents.

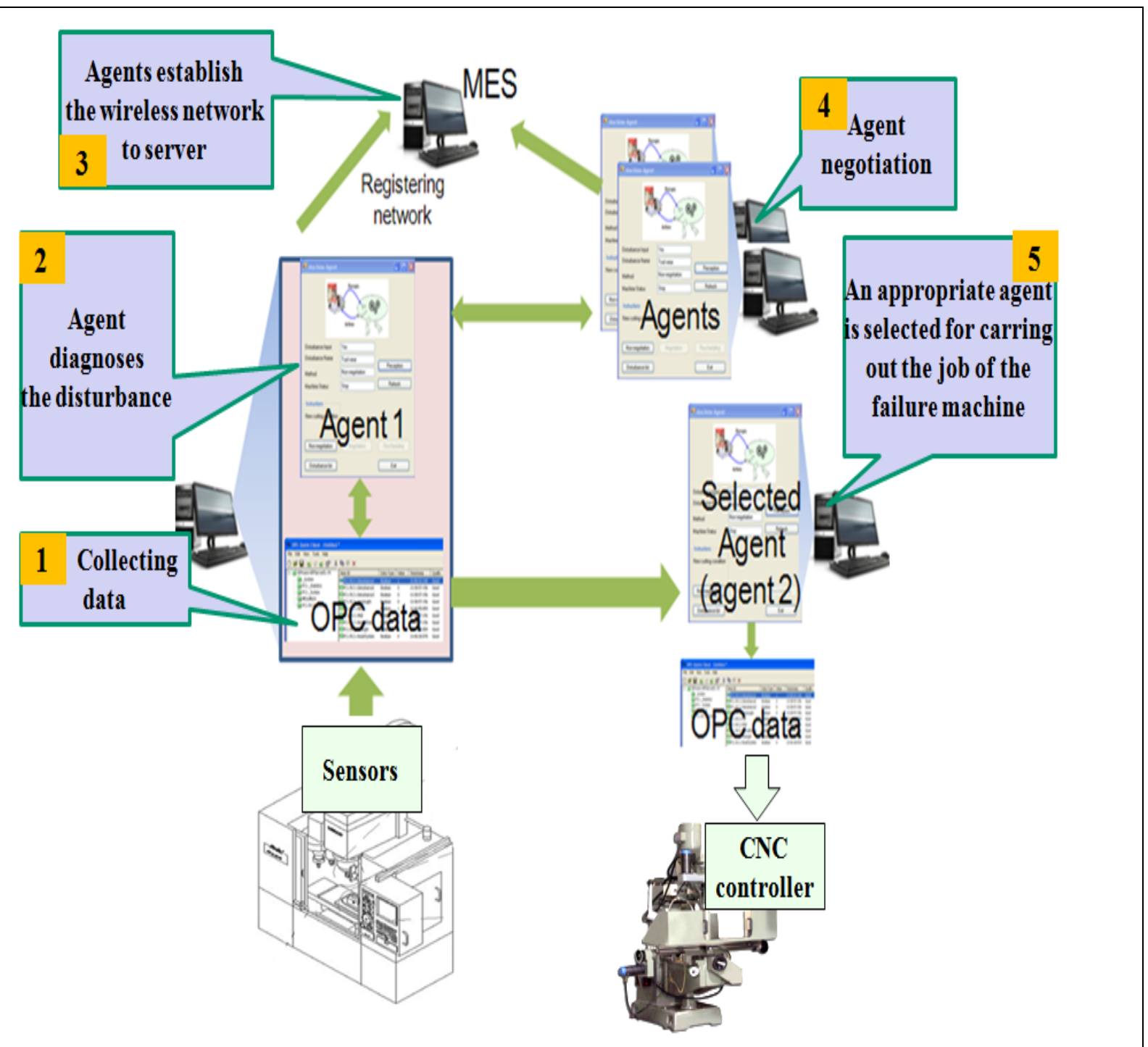

Fig. 30. Generating the optimal route among machines

\section{Conclusion}

Globalization, unpredictable markets, increased products customization and frequent changes in products, production technologies and manufacturing systems 
has become a complexity in today's manufacturing environment. One key strategy for coping with the evolution of this situation is to develop or apply an enable technology such as smart manufacturing. In this article, a new trend related to manufacturing technology is presented. The cognitive agents as well as advanced ICT infrastructure increase the system robustness by avoiding centralized control and show the potential of implementing autonomous behaviours by flexible ability in decision making. The manufacturing control system based on the cognitive agents has the enough ability to adapt autonomously to disturbances without upper-level aids or a total planning modification. On the other hand, the manufacturing control system equipped with the artificial cognitive capabilities meets the requirements of flexibility, adaptability and reliability. This technology enables the applicability of cognitive behaviours of human to overcome the disturbances within the machining system. It supports the fast response to disturbances without rescheduling. In the existing manufacturing systems, the dynamic rescheduling is done when the disturbances, such as the machine breakdown or malfunction of the robot, happen. Through the applications of the smart manufacturing in practice, the productivity of the machining system and the machining quality of products will be increased by using the selfadapting and self-optimizing processes, respectively. The development of a selfevolution mechanism for solving the new possible disturbances is considered as the future work of this research.

\section{References}

Abele E., Worn A. (2009) Reconfigurable machine tools and equipment, In: EIMaraghy, H.A (ed) Changeable and Reconfigurable Manufacturing Systems, Springer, pp. 111-125

Anghinolfi D., Boccalatte A., Grosso A., Paolucci M., Passadore A., Vecchiola C. (2007) A swarm intelligence method applied to manufacturing scheduling, Proceedings of WOA, 65-70

Brezocnik M., Balic J., Brezocnik Z. (2003) Emergence of intelligence in nextgeneration manufacturing systems, Robotics and Computer Integrated Manufacturing, 19: $55-63$

Brussel H.V. (1995), "Navigation" issues in intelligent autonomous systems, IAS-4 Conference Proceedings, pp.42-52

Choi B.K., Kim B.H. (2002) MES (manufacturing execution system) architecture for FMS compatible to ERP (enterprise planning system), International Journal of Computer Integrated Manufacturing, 15: 274-284

Christo C., Cardeira C. (2007), Trends in intelligent manufacturing systems, Proceedings of the IEEE International Symposium on Industrial Electronics, pp.32093214

Denkena B., Henning H., Lorenzen L.E. (2010), Genetics and intelligence: new approaches in production engineering, Production Management, 4: 65-73 
Denkena B., Mohring H.C., Litwinski K.M. (2008) Design of dynamic multi sensor systems, Production Engineering, 2: 327-331

Garg A., Gill P., Rathi P., Amardeep, Garg K.K. (2009) An insight into swarm intelligence, International Journal of Recent Trends in Engineering, 2(8): 42-44

Günther O.P., Kletti W., Kubach U. (2008) RFID in manufacturing, Springer

Kim D.H., Song J.Y., Lee S.H., Cha S.K. (2009) Development and evaluation of Zigbee node module for USN, International Journal of Precision Engineering and Manufacturing, 10: 53-57

Kletti J. (2007) Manufacturing execution system- MES, Springer

Landers R.G., Min B.K., Koren Y. (2001) Reconfigurable machine tools, CIRP Annals- Manufacturing Technology, 50(4): 269-274

Leitao P. (2004) An agile and adaptive holonic architecture for manufacturing control, $\mathrm{PhD}$ Dissertation

Leitao P. (2008), A bio-inspired solution for manufacturing control systems, In: Azevedo, A.(ed.) IFIP International Federation for Information Processing, Innovation in Manufacturing Networks, pp.303-314

Leitao P. (2009a), Agent-based distributed manufacturing control: A state-of-the-art survey, Engineering Applications of Artificial Intelligence, 22(7): 979-991

Leitao P. (2009b) Holonic rationale and bio-inspiration on design of complex emergent and evolvable systems, Transactions on Large-Scale Data and Knowledge -Centrered System I, Springer

Leitao P., Restivo F. (2002) Agent-based holonic production control, Proceedings of the 13th International Workshop on Database and Expert Systems Applications, 589596

Meyer, G.G., Framling, K. and Holmstrom, J. (2009), Intelligent Products: A survey, Computers in Industry 60, 137-148

Monostori L., Szelke E., Kadar B. (1998) Management of changes and disturbances in manufacturing systems, Annual Reviews in Control, 22: 85-97

Mori M., Fujishima M. (2009) Reconfigurable machine tools for a flexible manufacturing system, In: EIMaraghy, H.A (ed) Changeable and Reconfigurable Manufacturing Systems, Springer, pp. 101-109

Nakano T., Suda T. (2007) Applying biological principles to designs of network services, Applied Soft Computing, 7: 870-878

Pal S., Heyns P.S., Freyer B.H., Theron N.J., Pal S.K. (2011) Tool wear monitoring and selection of optimum cutting conditions with progressive tool wear effect and input uncertainties, J. Intel. Manuf., 22:491-504

Park H.S., Lee W.G. (2000) Agent-based shop control system under holonic manufacturing concept, Proc. of the 4th Korea-Russia International Symposium, 3: 116-121

Park H.S., Tran N.H. (2009) A concept of cognitive agent for controlling a manufacturing system, Proc. of the International Forum on Strategic Technology, pp. 95-100 
Park, H. S.: From Automation to Autonomy - a New Trend for Smart Manufacturing

Pritschow G., Wurst K.H., Kircher C., M. Seyfarth M. (2009) Control of reconfigurable machine tools, In: EIMaraghy, H.A (ed) Changeable and Reconfigurable Manufacturing Systems, Springer, pp. 71-100

Schmidt M., Wriggers F.S., Fisser F., Nyhuis P. (2008) Gentelligent Parts: A Decentralized Information System for Enterprises, IFIP International Federation for Information Processing, 254: 125-134

Serrano V., Fischer T. (2007) Collaborative innovation in ubiquitous systems, Journal of Intelligent Manufacturing, 18: 599-615.

Shirase K., Fujii S. (2009) Machine tool automation, Handbook of Automation, Springer, pp. 837-857

Tobias K., Werner H., Christian B. (2009) SOAR-based sequence control for a flexible assembly cell, Proceeding ETFA'09 Proceedings of the 14th IEEE international conference on Emerging technologies \& factory automation

Ueda K., Hatono I., Fujii N., Vaario J. (2000), Reinforcement learning approaches to biological manufacturing systems, Annals of the CIRP, 49(1): 343-346

Ueda K., Kito T., Fujii N. (2006), Modeling biological manufacturing system with bounded-rational agents, Annals of the CIRP 55(1): 469-472

Valckenaers P., Brussel H.V., Verstraete P., Germain B.S., Hadeli (2007) Schedule execution in autonomic manufacturing execution systems, Journal of Manufacturing Systems, 26: 75-84

Wu D., Thames J.L., Rosen D.W., Schaefer D. (2012) Towards a cloud-based design and manufacturing paradigm: Looking backward, looking forward, Proceedings of DETC conference

Wu K.H., Gastan E., Rodman M., Behrens B.A., Bach Fr.W., Gatzen H.H. (2010) Development and application of magnetic magnesium for data storage in gentelligent products, Journal of Magnetism and Magnetic Materials, 322: 1134-1136

Wu K.H., Traisigkhachol O., Gatzen H.H. (2008) A concept for magnetic data recording on genetically intelligent ("gentelligent") components, Physica status solidi. C. Current topics in solid state physics, 5(11): 3547-3550

Xiang W., LeeH.-P. (2008) Ant colony intelligence in multi-agent dynamic manufacturing scheduling, Engineering Applications of Artificial Intelligence, 21:7385

Yigit A.S., Ulsoy A.G. Application of nonlinear receptance coupling to dynamic stiffness evaluation for reconfigurable machine tools Yoshimi Ito (2008) Modular design for machine tools, McGraw-Hill

Zaeh M.F., Beetz M., Shea K., et al. (2009) The cognitive factory, In: EIMaraghy, H.A (ed) Changeable and reconfigurable manufacturing systems, Springer, pp. 355-371 Zhao X., Son Y. (2008), BDI-based human decision-making model in automated manufacturing systems, Int J Model Simul, 28(3):347-356 